IFIC/04-06

FTUV/04-0721

\title{
Quantum Loops in the Resonance Chiral Theory: The Vector Form Factor
}

\author{
I. Rosell, J.J. Sanz-Cillero and A. Pich \\ Departament de Fúsica Teòrica, IFIC, Universitat de València - CSIC \\ Apt. Correus 22085, E-46071 València, Spain
}

February 7, 2008

\begin{abstract}
We present a calculation of the Vector Form Factor at the next-to-leading order in the $1 / N_{C}$ expansion, within the framework of Resonance Chiral Theory. The calculation is performed in the chiral limit, and with two dynamical quark flavours. The ultraviolet behaviour of quantum loops involving virtual resonance propagators is analyzed, together with the kind of counterterms needed in the renormalization procedure. Using the lowest-order equations of motion, we show that only a few combinations of local couplings appear in the final result. The low-energy limit of our calculation reproduces the standard Chiral Perturbation Theory formula, allowing us to determine the resonance contribution to the chiral low-energy couplings, at the next-to-leading order in $1 / N_{C}$, keeping a full control of their renormalization scale dependence.
\end{abstract}




\section{Introduction}

We have at present an overwhelming experimental and theoretical evidence that the $S U(3)_{C}$ gauge theory correctly describes the hadronic world [1]. This makes QCD the established theory of the strong interactions. Nevertheless, its non-perturbative nature at long distances is still challenging our theoretical capabilities. Since the hadronization procedure is not understood, an effective field theory description [2] in terms of hadronic degrees of freedom is required in the low-energy regime.

Below the heavy quark thresholds, QCD is properly described considering only the light quarks, with masses much smaller than the dynamical QCD scale $\Lambda_{Q C D}$. One can then study the massless QCD case and consider the mass term as a perturbation. With $n_{f}$ massless quarks, QCD has a chiral $S U\left(n_{f}\right)_{R} \otimes S U\left(n_{f}\right)_{L}$ symmetry which is spontaneously broken to $S U\left(n_{f}\right)_{L+R}$. This generates $\left(n_{f}^{2}-1\right)$ Goldstone bosons, which can be identified with the multiplet of light pseudoscalars; their small masses being proportional to the explicit breaking of chiral symmetry generated by the quark masses. The Goldstone nature of the pseudoscalar bosons implies strong constraints on their interactions, which can be most easily analyzed on the basis of an effective Lagrangian organized as an expansion in powers of momenta (derivatives) and quark masses over the chiral symmetry breaking scale $\Lambda_{\chi} \simeq 4 \pi F_{\pi} \simeq 1.2$ $\mathrm{GeV}$. The resulting Goldstone effective field theory, Chiral Perturbation Theory $(\chi \mathrm{PT})[3-5]$, has achieved a remarkable success describing the low-energy dynamics of QCD $[6,7]$.

In the resonance region one must introduce a different effective field theory with explicit massive fields to describe the degrees of freedom associated with the mesonic resonances $[8,9]$. Although chiral symmetry still provides stringent dynamical constraints, the usual $\chi \mathrm{PT}$ power counting breaks down in the presence of higher energy scales. Therefore, one needs another expansion parameter to organize the effective Lagrangian. The limit of an infinite number of quark colours [10] turns out to be a very useful tool to understand many features of QCD and provides an alternative power counting to describe the meson interactions [11]. Taking $N_{C} \rightarrow \infty$, with $\alpha_{s} N_{C}$ fixed, there exists a systematic expansion of the $S U\left(N_{C}\right)$ gauge theory in powers of $1 / N_{C}$, which for $N_{C}=3$ provides a good quantitative approximation scheme to the hadronic world [12]. Assuming confinement, the strong dynamics at $N_{C} \rightarrow \infty$ is given by tree diagrams with infinite sums of hadron exchanges, which correspond to the tree approximation to some local effective Lagrangian. Hadronic loops generate corrections suppressed by factors of $1 / N_{C}$.

The short-distance properties of the underlying QCD dynamics impose strong constraints on the couplings of the hadronic effective theory $[9,11]$. The infinite sums of meson exchanges contributing to any given Green function should obey the right QCD behaviour $G(t) \sim t^{\omega}$ at large momenta. This requirement excludes resonance interactions with large number of derivatives, explaining the phenomenological success of the usual lowest-order (in derivatives) approximations. Moreover, it implies stringent correlations among those resonance couplings 
associated with the highest powers of momenta. The usual $\chi \mathrm{PT}$ expansion is of course recovered at very low energies, when the resonance Green functions are expanded in powers of momenta over the resonance mass scale.

In spite of its many dynamical simplifications, QCD at $N_{C} \rightarrow \infty$ is still a very involved theory and some approximations are called for. Usually one cuts the infinite tower of resonance exchanges to a finite number, taking only into account those meson states which are relevant at the physical energy scale. This is meaningful since the contributions from highermass states are suppressed by their corresponding propagators. However, it introduces back a momentum expansion regulated by inverse powers of the heavier resonance masses which have been integrated out. The problem can be formally avoided taking the limit $M_{R} \rightarrow \infty$ for all resonance states not included in the effective theory. This gives a well-defined approximation with a clear physical meaning: one is assuming that the QCD short-distance operator product expansion provides an acceptable description at energies above the last included mesonic state. The imposed short-distance constraints are nothing else than matching conditions between the low-energy effective field theory and the underlying QCD dynamics.

The most drastic and simplest scheme is the so-called Single Resonance Approximation, which only considers the contributions from the lightest meson with any given quantum numbers $[8,9,13,14]$. The short-distance QCD constraints determine in this case all hadronic parameters in terms of the pion decay constant $F_{\pi}$ and the two masses of the vector and scalar multiplets, $M_{V}$ and $M_{S}$ [11]. This gives a very successful description at energies below the scale of the second resonance multiplets. Since there is an infinite number of Green functions, it is clearly not possible to satisfy all matching conditions within the single resonance approximation. A useful generalization is the Minimal Hadronic Ansatz, which keeps the minimum number of resonances compatible with all known short-distance constraints for the problem at hand [15]. The resonance contributions to some $\mathcal{O}\left(p^{6}\right) \chi \mathrm{PT}$ couplings have been already analyzed in this way, by studying an appropriate set of three-point functions [16-18].

The $N_{C} \rightarrow \infty$ limit of the resonance chiral theory $(\mathrm{R} \chi \mathrm{T})$ has been investigated in many works $[8,9,11,13-18]$ and a very successful leading order phenomenology already exists [11]. However, we are still lacking a systematic procedure to incorporate next-to-leading contributions in the $1 / N_{C}$ counting. Up to now, the effort has concentrated in pinning down the most relevant subleading effects, such as the resonance widths which regulate the corresponding poles in the meson propagators [19-21], or the role of final state interactions in the physical amplitudes [19-27]. These unitarity corrections are generated through Goldstone loops and therefore are suppressed by $1 / N_{C}$ factors; nevertheless they may be strongly enhanced by large infrared logarithms. The combined constraints of analyticity and unitarity make possible to perform appropriate resummations of chiral logarithms, which describe the leading $1 / N_{C}$ corrections in the resonance region.

Quantum loops including virtual resonance propagators are a major technical challenge which has never been properly addressed. A first step in this direction was the study of reso- 
nance loop contributions to the running of the $\chi \mathrm{PT}$ coupling $L_{10}(\mu)$, performed in Ref. [28], which however didn't attempt any analysis of the induced ultraviolet divergences and their corresponding renormalization. ${ }^{1}$ Clearly, at the one-loop level the massive states present in $\mathrm{R} \chi \mathrm{T}$ generate all kind of ultraviolet problems which are not yet understood. A naive chiral power counting indicates that the renormalization procedure will require higher dimensional counterterms, which presumably could generate a problematic behaviour at large momenta. Therefore, it will be necessary to perform a careful investigation of the constraints implied by the short-distance properties of QCD at the next-to-leading order in $1 / N_{C}$.

A formal renormalization of $\mathrm{R} \chi \mathrm{T}$ at the one-loop level appears to be a very involved task, which requires the prior analysis of several technical ingredients [34]. In order to gain some understanding on the ultraviolet behaviour, it seems worth to perform first some explicit oneloop calculations of well chosen physical amplitudes. In the following, we present a detailed investigation of the pion vector form factor $(\mathrm{VFF})$ at the next-to-leading order (NLO) in the $1 / N_{C}$ expansion. This observable is defined through the two-Goldstone matrix element of the vector current:

$$
\left\langle\pi^{+}\left(p_{1}\right) \pi^{-}\left(p_{2}\right)\left|\frac{1}{2}\left(\bar{u} \gamma^{\mu} u-\bar{d} \gamma^{\mu} d\right)\right| 0\right\rangle=\mathcal{F}\left(q^{2}\right)\left(p_{1}-p_{2}\right)^{\mu},
$$

where $q^{\mu} \equiv\left(p_{1}+p_{2}\right)^{\mu}$. At very low energies, the $\operatorname{VFF} \mathcal{F}\left(q^{2}\right)$ has been studied within the $\chi \mathrm{PT}$ framework up to $\mathcal{O}\left(p^{6}\right)[35-37]$. R $\chi \mathrm{T}$ and the $1 / N_{C}$ expansion have been also used to determine $\mathcal{F}\left(q^{2}\right)$ at the $\rho$ meson peak, including appropriate resummations of subleading infrared logarithms [19-22].

We will simplify the calculation working in the two flavour theory and taking the massless quark limit. Therefore, we will assume a chiral $U(2)_{L} \otimes U(2)_{R}$ symmetry group. The small effects induced by the $U(1)_{A}$ anomaly will be neglected, because they are not going to be relevant in our discussion. The isosinglet pseudoscalar can only appear within loops, and the numerical correction generated by its non-zero mass could be taken into account in a straightforward way, together with the finite quark mass effects which we are ignoring.

In the next section we will briefly describe the $\mathrm{R} \chi \mathrm{T}$ Lagrangian. We will adopt the single resonance approximation and will only consider the minimal set of resonance couplings (linear in the resonance fields) introduced in ref. [8], supplemented with those counterterms required by the renormalization procedure. The renormalization of the relevant one-particleirreducible Feynman diagrams will be discussed in section 3 and the final results of our calculation will be collected in section 4 . Sections 5 and 6 analyze the behaviour of the computed vector form factor at low and high energies, respectively. We will finally summarize our findings in section [7. Some technical details have been relegated to the appendices.

\footnotetext{
${ }^{1}$ Quantum loops involving massive states have been only analyzed within explicit models with additional symmetries. For instance, the gauge structure advocated in the so called "Hidden Local Symmetry" description of vector resonances [29] implies a much simpler ultraviolet behaviour [30]. Chiral loop corrections to some vector resonance parameters have been also studied [31,32] within the context of "Heavy Vector Meson $\chi \mathrm{PT} "$ [33], which adopts the $M_{R} \rightarrow \infty$ limit to guarantee a good chiral power counting.
} 


\section{The Lagrangian of Resonance Chiral Theory}

We are going to work within a $U(2)_{L} \otimes U(2)_{R}$ chiral theory, containing a multiplet of 4 pseudoscalar Goldstone bosons,

$$
\Phi=\left(\begin{array}{cc}
\frac{1}{\sqrt{2}} \pi^{0}+\frac{1}{\sqrt{2}} \eta_{0} & \pi^{+} \\
\pi^{-} & -\frac{1}{\sqrt{2}} \pi^{0}+\frac{1}{\sqrt{2}} \eta_{0}
\end{array}\right),
$$

parameterized through the unitary matrix $u(\Phi)=\exp \{i \Phi /(\sqrt{2} F)\}$. The Goldstones couple to massive $U(2)$ multiplets of the type $V\left(1^{--}\right), A\left(1^{++}\right), S\left(0^{++}\right)$and $P\left(0^{-+}\right)$, with a field content analogous to the one indicated in (2).

Our starting point is the $\mathrm{R} \chi \mathrm{T}$ Lagrangian $\mathcal{L}(u, V, A, S, P)$ introduced in Ref. [8]. It contains the $\mathcal{O}\left(p^{2}\right) \chi \mathrm{PT}$ Lagrangian $[4,5]$,

$$
\mathcal{L}_{2 \chi}=\frac{F^{2}}{4}\left\langle u_{\mu} u^{\mu}+\chi_{+}\right\rangle
$$

the kinetic resonance Lagrangians,

$$
\begin{aligned}
& \mathcal{L}_{2 Z}(R=V, A)=-\frac{1}{2}\left\langle\nabla^{\lambda} R_{\lambda \mu} \nabla_{\nu} R^{\nu \mu}-\frac{1}{2} M_{R}^{2} R_{\mu \nu} R^{\mu \nu}\right\rangle, \\
& \mathcal{L}_{2 Z}(R=S, P)=\frac{1}{2}\left\langle\nabla^{\mu} R \nabla_{\mu} R-M_{R}^{2} R^{2}\right\rangle,
\end{aligned}
$$

and $\mathcal{O}\left(p^{2}\right)$ interactions linear in the resonance fields:

$$
\begin{aligned}
\mathcal{L}_{2 V}\left[V\left(1^{--}\right)\right] & =\frac{F_{V}}{2 \sqrt{2}}\left\langle V_{\mu \nu} f_{+}^{\mu \nu}\right\rangle+\frac{i G_{V}}{2 \sqrt{2}}\left\langle V_{\mu \nu}\left[u^{\mu}, u^{\nu}\right]\right\rangle \\
\mathcal{L}_{2 A}\left[A\left(1^{++}\right)\right] & =\frac{F_{A}}{2 \sqrt{2}}\left\langle A_{\mu \nu} f_{-}^{\mu \nu}\right\rangle \\
\mathcal{L}_{2 S}\left[S\left(0^{++}\right)\right] & =c_{d}\left\langle S u_{\mu} u^{\mu}\right\rangle+c_{m}\left\langle S \chi_{+}\right\rangle, \\
\mathcal{L}_{2 P}\left[P\left(0^{-+}\right)\right] & =i d_{m}\left\langle P \chi_{-}\right\rangle .
\end{aligned}
$$

The brackets $\langle\ldots\rangle$ denote a trace of the corresponding flavour matrices, and the different chiral tensors follow the notation defined in Ref. [8]. They include external vector, axial-vector, scalar and pseudoscalar sources $\left(v^{\mu}, a^{\mu}, s, p\right)$ to generate the corresponding Green functions. Following this reference, we describe the vector and axial-vector resonances with the antisymmetric field formalism. In the chiral limit and neglecting external scalar or pseudoscalar sources $\chi_{ \pm}=0$.

The Lagrangian $\mathcal{L}(u, V, A, S, P)$ obeys the correct $N_{C}$ counting rules. The different fields and the masses and momenta are all of them $\mathcal{O}(1)$, whereas all couplings $\left(F, F_{V}, G_{V}, F_{A}\right.$, 
$c_{d}, c_{m}$ and $\left.d_{m}\right)$ are of $\mathcal{O}\left(\sqrt{N_{C}}\right)$. In the limit $N_{C} \rightarrow \infty$, one can determine all parameters in terms of $F, M_{V}$ and $M_{S}$ [11]. The short-distance QCD behaviour of the vector, axialvector [9] and scalar [24] form factors, together with the constraints implied [9,11,14] by the superconvergence properties of the $v v-a a$ [38] and $s s-p p$ two-point functions at large momenta, imply the relations [11]:

$$
\begin{aligned}
& \frac{F_{V}}{\sqrt{2}}=\sqrt{2} G_{V}=F_{A}=2 c_{m}=2 c_{d}=2 \sqrt{2} d_{m}=F, \\
& M_{A}=\sqrt{2} M_{V} \quad, \quad M_{P}=\sqrt{2} M_{S}(1-\delta)^{1 / 2} .
\end{aligned}
$$

The last identity involves a small correction $\delta \approx 3 \pi \alpha_{s} F^{2} / M_{S}^{2} \sim 0.08 \alpha_{s}$, which can be neglected together with the tiny effects from light quark masses.

\subsection{Subleading Lagrangian}

The one loop calculation of the VFF with the previous Lagrangian generates ultraviolet divergences which require counterterms with a higher number of derivatives. We will only include the minimal set of chiral structures needed to renormalize the VFF calculation. We expect their corresponding couplings to be subleading in the $1 / N_{C}$ expansion, since they are associated with quantum loop corrections.

We need to include the following $\mathcal{O}\left(p^{4}\right)$ and $\mathcal{O}\left(p^{6}\right)$ Goldstone interactions:

$$
\begin{gathered}
\widetilde{\mathcal{L}}_{4 \chi}=\frac{i \widetilde{\ell}_{6}}{4}\left\langle f_{+}^{\mu \nu}\left[u_{\mu}, u_{\nu}\right]\right\rangle-\tilde{\ell}_{12}\left\langle\nabla^{\mu} u_{\mu} \nabla^{\nu} u_{\nu}\right\rangle, \\
\widetilde{\mathcal{L}}_{6 \chi}=i \widetilde{c}_{51}\left\langle\nabla^{\rho} f_{+}^{\mu \nu}\left[h_{\mu \rho}, u_{\nu}\right]\right\rangle+i \widetilde{c}_{53}\left\langle\nabla_{\mu} f_{+}^{\mu \nu}\left[h_{\nu \rho}, u^{\rho}\right]\right\rangle .
\end{gathered}
$$

Chiral invariance forces these terms to have structures contained in the corresponding $\chi \mathrm{PT}$ Lagrangians $[4,39]$. We use a tilde to denote the $\mathrm{R} \chi \mathrm{T}$ couplings in (8) and (9), which are different to the ones with the same names (without tilde) in $\chi \mathrm{PT}$. For instance, the $\chi \mathrm{PT}$ coupling $\ell_{6}$ ( $L_{9}$ in the three flavour case) is dominated by a contribution from vector-meson exchange and is of $\mathcal{O}\left(N_{C}\right)$, while the corresponding $\mathrm{R} \chi \mathrm{T}$ coupling $\tilde{\ell}_{6}$ does not contain this contribution and is of $\mathcal{O}(1)$.

The $\mathcal{O}\left(p^{4}\right)$ term containing the structure $\nabla^{\mu} u_{\mu}$ does not contribute to the tree-level calculation of the VFF; nevertheless, it is needed to renormalize the Goldstone self-energies. At $\mathcal{O}\left(p^{6}\right)$, only the combination of couplings $\widetilde{r}_{V 2} \equiv 4 F^{2}\left(\widetilde{c}_{53}-\widetilde{c}_{51}\right)$ is going to be relevant for the VFF [39]. Including the Lagrangians (8) and (9), the tree level calculation of the VFF gives the result:

$$
\mathcal{F}\left(q^{2}\right)=1+\frac{F_{V} G_{V}}{F^{2}} \frac{q^{2}}{M_{V}^{2}-q^{2}}-\tilde{\ell}_{6} \frac{q^{2}}{F^{2}}+\widetilde{r}_{V 2} \frac{q^{4}}{F^{4}}
$$



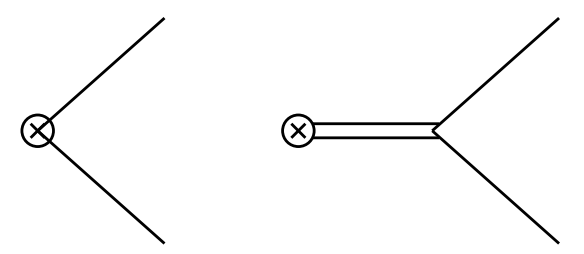

Figure 1: Leading order contributions to the VFF.

The requirement that the VFF should vanish at $q^{2} \rightarrow \infty$ implies the following conditions at leading order in $1 / N_{C}$ :

$$
F_{V} G_{V}=F^{2} \quad, \quad \tilde{\ell}_{6}=0 \quad, \quad \widetilde{r}_{V 2} \equiv 4 F^{2}\left(\widetilde{c}_{53}-\widetilde{c}_{51}\right)=0 .
$$

Therefore, the couplings $\tilde{\ell}_{6} / F^{2}$ and $\widetilde{r}_{V 2} / F^{4}$ are of subleading order in the $1 / N_{C}$ expansion, i.e. $\mathcal{O}\left(1 / N_{C}\right)$, as expected on pure dimensional grounds.

The renormalization of Green functions including resonance fields forces the presence of the following additional counterterms:

$$
\begin{gathered}
\mathcal{L}_{4 Z}=\frac{X_{Z_{1}}}{2}\left\langle\nabla^{2} V^{\mu \nu}\left\{\nabla_{\nu}, \nabla^{\sigma}\right\} V_{\mu \sigma}\right\rangle+\frac{X_{Z_{2}}}{4}\left\langle\left\{\nabla_{\nu}, \nabla_{\alpha}\right\} V^{\mu \nu}\left\{\nabla^{\sigma}, \nabla^{\alpha}\right\} V_{\mu \sigma}\right\rangle \\
+\frac{X_{Z_{3}}}{4}\left\langle\left\{\nabla^{\sigma}, \nabla^{\alpha}\right\} V^{\mu \nu}\left\{\nabla_{\nu}, \nabla_{\alpha}\right\} V_{\mu \sigma}\right\rangle, \\
\mathcal{L}_{4 F}=X_{F_{1}}\left\langle V_{\mu \nu} \nabla^{2} f_{+}^{\mu \nu}\right\rangle+X_{F_{2}}\left\langle V_{\mu \nu}\left\{\nabla^{\mu}, \nabla_{\alpha}\right\} f_{+}^{\alpha \nu}\right\rangle, \\
\mathcal{L}_{4 G}=i X_{G_{1}}\left\langle\left\{\nabla^{\alpha}, \nabla_{\mu}\right\} V^{\mu \nu}\left[u_{\nu}, u_{\alpha}\right]\right\rangle+i X_{G_{2}}\left\langle V^{\mu \nu}\left[h_{\alpha \mu}, h_{\nu}^{\alpha}\right]\right\rangle .
\end{gathered}
$$

The quadratic Lagrangian $\mathcal{L}_{4 Z}$ is needed to renormalize the vector self-energy. Actually, only the sum of couplings $X_{Z} \equiv X_{Z_{1}}+X_{Z_{2}}+X_{Z_{3}}$ is relevant for this purpose. The renormalization of the $v-V$ two-point function involves the sum of $\mathcal{L}_{4 F}$ couplings $X_{F} \equiv$ $X_{F_{1}}+X_{F_{2}}$. Finally, the three-point function with one external vector resonance and two Goldstone legs is renormalized by $\mathcal{L}_{4 G}$ through the combination $X_{G} \equiv X_{G_{2}}-\frac{1}{2} X_{G_{1}}$. The dimensions of the couplings are $\left[X_{Z}\right]=E^{-2}$ and $\left[X_{F}\right]=\left[X_{G}\right]=E^{-1}$.

At the NLO in $1 / N_{C}$, these counterterm Lagrangians only contribute through tree-level diagrams. One can then use the leading order equation of motion (EOM)

$$
\nabla^{\mu} \nabla_{\rho} V^{\rho \nu}-\nabla^{\nu} \nabla_{\rho} V^{\rho \mu}=-M_{V}^{2} V^{\mu \nu}-\frac{F_{V}}{\sqrt{2}} f_{+}^{\mu \nu}-\frac{i G_{V}}{\sqrt{2}}\left[u^{\mu}, u^{\nu}\right]
$$


to reduce the number of relevant operators. The Lagrangians (12), (13) and (14) take then the equivalent forms:

$$
\begin{gathered}
\mathcal{L}_{4 Z}^{\mathrm{EOM}}=\frac{X_{Z} M_{V}^{4}}{2}\left\langle V^{\mu \nu} V_{\mu \nu}\right\rangle+\frac{X_{Z} M_{V}^{2} F_{V}}{\sqrt{2}}\left\langle V_{\mu \nu} f_{+}^{\mu \nu}\right\rangle+\frac{i X_{Z} M_{V}^{2} G_{V}}{\sqrt{2}}\left\langle V_{\mu \nu}\left[u^{\mu}, u^{\nu}\right]\right\rangle \\
+\frac{i X_{Z} F_{V} G_{V}}{2}\left\langle f_{+}^{\mu \nu}\left[u_{\mu}, u_{\nu}\right]\right\rangle+\cdots, \\
\mathcal{L}_{4 F}^{\mathrm{EOM}}=-X_{F} M_{V}^{2}\left\langle V_{\mu \nu} f_{+}^{\mu \nu}\right\rangle-\frac{i X_{F} G_{V}}{\sqrt{2}}\left\langle f_{+}^{\mu \nu}\left[u_{\mu}, u_{\nu}\right]\right\rangle+\cdots, \\
\mathcal{L}_{4 G}^{\mathrm{EOM}}=-2 i X_{G} M_{V}^{2}\left\langle V^{\alpha \nu}\left[u_{\alpha}, u_{\nu}\right]\right\rangle-i \sqrt{2} X_{G} F_{V}\left\langle f_{+}^{\mu \nu}\left[u_{\mu}, u_{\nu}\right]\right\rangle+\cdots,
\end{gathered}
$$

where the dots denote other terms which are not relevant for the VFF calculation at this order. The derivatives acting on the vector resonance fields have been traded by the heavy mass scale $M_{V}$ and/or derivatives acting on the Goldstone fields, giving rise to the usual tensor structures of the $\chi \mathrm{PT}$ Lagrangian. Therefore, the effect of the counterterm Lagrangians $\mathcal{L}_{4 Z}$, $\mathcal{L}_{4 F}$ and $\mathcal{L}_{4 G}$ is just equivalent to the following shift in the couplings at the next-to-leading order in $1 / N_{C}$ :

$$
\begin{aligned}
\tilde{\ell}_{6}^{\mathrm{eff}} & =\tilde{\ell}_{6}+2 X_{Z} F_{V} G_{V}-2 \sqrt{2} X_{F} G_{V}-4 \sqrt{2} X_{G} F_{V}, \\
F_{V}^{\mathrm{eff}} & =F_{V}+2 X_{Z} M_{V}^{2} F_{V}-2 \sqrt{2} X_{F} M_{V}^{2} \\
G_{V}^{\mathrm{eff}} & =G_{V}+2 X_{Z} M_{V}^{2} G_{V}-4 \sqrt{2} X_{G} M_{V}^{2}, \\
\left(M_{V}^{2}\right)^{\mathrm{eff}} & =M_{V}^{2}+2 X_{Z} M_{V}^{4}, \\
\widetilde{r}_{V 2}^{\mathrm{eff}} & =\widetilde{r}_{V 2} .
\end{aligned}
$$

Thus, since $\tilde{\ell}_{6}^{\text {eff }} \sim \tilde{\ell}_{6} \sim\left(M_{V}^{2}\right)^{\text {eff }} \sim M_{V}^{2} \sim \mathcal{O}(1)$ and $F_{V}^{\text {eff }} \sim F_{V} \sim G_{V}^{\text {eff }} \sim G_{V} \sim \mathcal{O}\left(\sqrt{N_{C}}\right)$, a consistent $1 / N_{C}$ counting requires that $X_{G}$ and $X_{F}$ are of $\mathcal{O}\left(1 / \sqrt{N_{C}}\right)$ and $X_{Z}$ of $\mathcal{O}\left(1 / N_{C}\right)$.

\section{Renormalization of Quantum Loops}

The renormalization procedure follows very systematic and precise steps in any well defined field theory. First of all, the two-point Green functions must be renormalized. Later the three-point Green functions and so on. For the VFF up to NLO in $1 / N_{C}$ only the two- and three-point Green functions will contribute. The corresponding renormalizations for the one particle irreducible diagrams (1PI) at one loop are given in the next subsections. 
We will adopt the $\overline{M S}-1$ scheme, usually employed in $\chi \mathrm{PT}$ calculations, where one subtracts the divergent constant

$$
\lambda_{\infty}=\frac{2 \mu^{d-4}}{d-4}+\gamma_{E}-\ln 4 \pi-1
$$

However, we will impose the on-shell condition to renormalize the pion self-energy. This simplifies the calculation of physical amplitudes with external pions. Since we work in the massless quark limit, the Goldstone tadpoles will not give any contribution. The precise definition of the relevant Feynman integrals with one, two and three propagators and some useful antisymmetric formalism technology are relegated to appendices A and B.

\subsection{Pion self-energy}
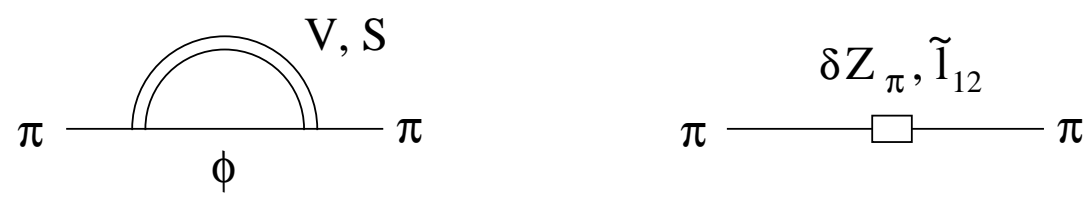

Figure 2: One-loop diagrams and local contributions to the pion self-energy.

The diagrams contributing to the pion propagator are shown in Fig. 2, The kinetic Lagrangians $\mathcal{L}_{2 Z}(R)$ in Eq. (44) generate additional tadpole topologies with one resonance propagator, but they are identically zero even with massive pions. The divergences of $\mathcal{O}\left(p^{2}\right)$ are reabsorbed through the wave-function renormalization $\pi^{(B)}=\left(1+\delta Z_{\pi}\right)^{\frac{1}{2}} \pi^{(r)}$, being $\pi^{(B)}$ and $\pi^{(r)}$ the bare and renormalized pion fields respectively. In the on-shell scheme,

$$
\delta Z_{\pi}=-\frac{2 G_{V}^{2}}{F^{2}} \frac{3 M_{V}^{2}}{16 \pi^{2} F^{2}}\left\{\lambda_{\infty}+\ln \frac{M_{V}^{2}}{\mu^{2}}+\frac{1}{6}\right\}+\frac{4 c_{d}^{2}}{F^{2}} \frac{M_{S}^{2}}{16 \pi^{2} F^{2}}\left\{\lambda_{\infty}+\ln \frac{M_{S}^{2}}{\mu^{2}}-\frac{1}{2}\right\} .
$$

There are also divergences of $\mathcal{O}\left(p^{4}\right)$ which renormalize one of the couplings in $\widetilde{\mathcal{L}}_{4 \chi}$ :

$$
\tilde{\ell}_{12} \equiv \tilde{\ell}_{12}^{r}(\mu)+\delta \tilde{\ell}_{12}(\mu) \quad ; \quad \delta \tilde{\ell}_{12}(\mu)=-\frac{G_{V}^{2}+2 c_{d}^{2}}{F^{2}} \frac{\lambda_{\infty}}{32 \pi^{2}} .
$$

The renormalized pion self-energy takes the form

$$
\begin{gathered}
-i \Sigma_{\pi}^{r}\left(p^{2}\right)=-i \frac{p^{4}}{16 \pi^{2} F^{2}}\left\{64 \pi^{2} \widetilde{\ell}_{12}^{r}(\mu)+\frac{2 G_{V}^{2}}{F^{2}}\left[\ln \frac{M_{V}^{2}}{\mu^{2}}+\phi\left(\frac{p^{2}}{M_{V}^{2}}\right)\right]\right. \\
\left.+\frac{4 c_{d}^{2}}{F^{2}}\left[\ln \frac{M_{S}^{2}}{\mu^{2}}+\phi\left(\frac{p^{2}}{M_{S}^{2}}\right)\right]\right\},
\end{gathered}
$$


where the function

$$
\phi(x)=\left(1-\frac{1}{x}\right)^{2}\left[\left(1-\frac{1}{x}\right) \ln (1-x)-1+\frac{x}{2}\right]=-(1-x)^{2} \sum_{n=0}^{\infty} \frac{x^{n}}{(n+2)(n+3)}
$$

contains finite and scale-independent contributions.

\subsection{Rho self-energy}
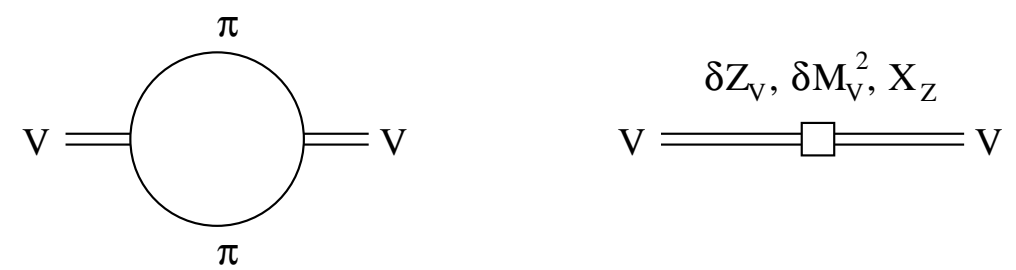

Figure 3: Rho self-energy.

The one loop $\rho$ self-energy contains only an $\mathcal{O}\left(p^{4}\right)$ divergence, which renormalizes the coupling $X_{Z}$ of the NLO resonance Lagrangian:

$$
X_{Z} \equiv X_{Z}^{r}(\mu)+\delta X_{Z}(\mu) \quad ; \quad \delta X_{Z}(\mu)=-\frac{2 G_{V}^{2}}{F^{2}} \frac{\lambda_{\infty}}{192 \pi^{2} F^{2}} .
$$

Thus, the vector mass and wave-function are not renormalized:

$$
\delta M_{V}^{2}=0 \quad ; \quad \delta Z_{V}=0 .
$$

The renormalized $\rho$ self-energy then becomes:

$$
-i \Sigma_{V}^{r}(q)^{\mu \nu, \rho \sigma}=-\frac{i}{2} \mathcal{A}^{\mu \nu, \rho \sigma}(q) \Sigma_{V}^{r}\left(q^{2}\right),
$$

where the antisymmetric tensor structure $\mathcal{A}^{\mu \nu, \rho \sigma}(q)$ is defined in appendix B and

$$
\Sigma_{V}^{r}\left(q^{2}\right)=-q^{4}\left\{2 X_{Z}^{r}(\mu)-\frac{2 G_{V}^{2}}{F^{2}} \frac{1}{F^{2}}\left[\frac{1}{6} \hat{B}_{0}\left(q^{2} / \mu^{2}\right)+\frac{1}{144 \pi^{2}}\right]\right\} .
$$

\section{3 $\left\langle v^{\mu} V^{\rho \sigma}\right\rangle$ 1PI vertex}

The 1PI amputated diagrams (at NLO) connecting an external vector quark current to an

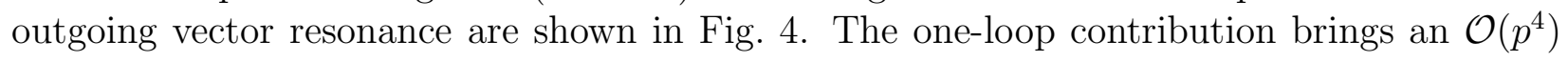
divergence which gets reabsorbed through the following renormalization of the coupling $X_{F}$ :

$$
X_{F} \equiv X_{F}^{r}(\mu)+\delta X_{F}(\mu) \quad ; \quad \delta X_{F}(\mu)=-\frac{\sqrt{2} G_{V}}{F} \frac{\lambda_{\infty}}{192 \pi^{2} F} .
$$



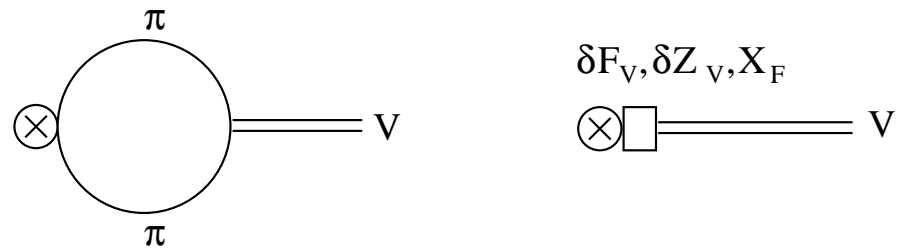

Figure 4: Diagrams contributing to the $\left\langle v^{\mu} V^{\rho \sigma}\right\rangle$ Green function at NLO in $1 / N_{C}$.

Since there are no divergences of $\mathcal{O}\left(p^{2}\right)$, the lowest-order coupling $F_{V}$ remains unchanged:

$$
\delta F_{V}=0 .
$$

The renormalized vertex function takes the form

$$
i \Phi(q)^{\mu, \rho \sigma}=-i \mathcal{I}_{\alpha \beta}^{\rho \sigma} q^{\alpha} g^{\mu \beta}\left\{F_{V}-2 \sqrt{2} X_{F}^{r}(\mu) q^{2}+\frac{2 G_{V}}{F^{2}} q^{2}\left[\frac{1}{6} \hat{B}_{0}\left(q^{2} / \mu^{2}\right)+\frac{1}{144 \pi^{2}}\right]\right\},
$$

where the first term is the leading order contribution. The massless two-point function $\hat{B}_{0}\left(q^{2} / \mu^{2}\right)$ is defined in appendix A and the antisymmetric tensor structure $\mathcal{I}_{\alpha \beta}^{\rho \sigma}$ in appendix B.

\section{$3.4\left\langle V_{\mu \nu} \pi \pi\right\rangle$ 1PI vertex}

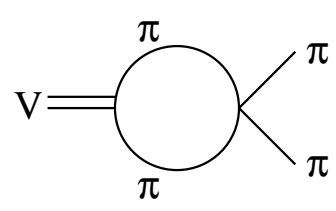

a)

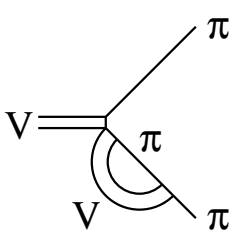

b)

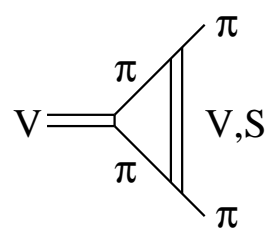

c)

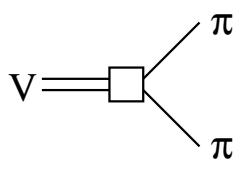

$\delta \mathrm{G}_{\mathrm{V}}, \delta \mathrm{Z}_{\pi}, \delta \mathrm{Z}_{\mathrm{V}}, \mathrm{X}_{\mathrm{G}}$

d)

Figure 5: NLO diagrams contributing to the three-point Green function $V^{\mu \nu} \rightarrow \pi \pi$.

The 1PI amputated diagrams connecting a vector resonance with two outgoing pions at NLO in $1 / N_{C}$ are shown in Fig. 5. The loop diagrams generate $\mathcal{O}\left(p^{2}\right)$ and $\mathcal{O}\left(p^{4}\right)$ divergences, which renormalize the couplings $G_{V}$ and $X_{G}$, respectively:

$$
G_{V} \equiv G_{V}^{r}(\mu)+\delta G_{V}(\mu) \quad ; \quad \delta G_{V}(\mu)=G_{V}\left[3 M_{V}^{2}\left(\frac{2 G_{V}^{2}}{F^{2}}-\frac{1}{2}\right)-M_{S}^{2} \frac{4 c_{d}^{2}}{F^{2}}\right] \frac{\lambda_{\infty}}{16 \pi^{2} F^{2}}
$$




$$
X_{G} \equiv X_{G}^{r}(\mu)+\delta X_{G}(\mu) \quad ; \quad \delta X_{G}(\mu)=\frac{\sqrt{2} G_{V}}{F}\left[\frac{2 G_{V}^{2}}{F^{2}}+\frac{4 c_{d}^{2}}{F^{2}}-2\right] \frac{\lambda_{\infty}}{1536 \pi^{2} F} .
$$

The wave-function renormalization of the external vector and pion legs amounts to a global factor $\left(\delta Z_{\pi}+\frac{1}{2} \delta Z_{V}\right)$ multiplying the lowest-order contribution $\left(\delta Z_{V}=0\right.$ at NLO). Taking this into account, one finally gets the finite vertex function

$$
i \Gamma_{\mu \nu}^{r}\left(p_{1}, p_{2}\right)=\mathcal{I}_{\mu \nu}^{\alpha \beta} q_{\alpha}\left(p_{1}-p_{2}\right)_{\beta} \frac{1}{F^{2}}\left\{G_{V}^{r}(\mu)\left[1-\Delta \Gamma\left(q^{2}, \mu^{2}\right)\right]-4 \sqrt{2} X_{G}^{r}(\mu) q^{2}\right\},
$$

where

$$
\begin{aligned}
\Delta \Gamma\left(q^{2}, \mu^{2}\right)=\frac{1}{F^{2}} & \left\{\hat{B}_{0}\left(q^{2} / \mu^{2}\right)\left[\frac{2 G_{V}^{2}}{F^{2}}\left(\frac{M_{V}^{4}}{q^{2}}+2 M_{V}^{2}+\frac{q^{2}}{12}\right)+\frac{4 c_{d}^{2}}{F^{2}}\left(\frac{M_{S}^{4}}{q^{2}}+\frac{q^{2}}{12}\right)-\frac{q^{2}}{6}\right]\right. \\
& +\frac{M_{V}^{2}}{16 \pi^{2}} \ln \frac{M_{V}^{2}}{\mu^{2}}\left[\frac{2 G_{V}^{2}}{F^{2}}\left(\frac{M_{V}^{2}}{q^{2}}+5\right)-\frac{3}{2}\right]+\frac{M_{S}^{2}}{16 \pi^{2}} \ln \frac{M_{S}^{2}}{\mu^{2}} \frac{4 c_{d}^{2}}{F^{2}}\left(\frac{M_{S}^{2}}{q^{2}}-1\right) \\
& +\frac{M_{V}^{2}}{64 \pi^{2}}\left[3 \frac{2 G_{V}^{2}}{F^{2}}-1\right]+\frac{3 M_{S}^{2}}{64 \pi^{2}} \frac{4 c_{d}^{2}}{F^{2}}+\frac{q^{2}}{288 \pi^{2}}\left[\frac{2 G_{V}^{2}}{F^{2}}+\frac{4 c_{d}^{2}}{F^{2}}-2\right] \\
& +\frac{2 G_{V}^{2}}{F^{2}} C_{0}\left(q^{2}, 0,0, M_{V}^{2}\right)\left[\frac{M_{V}^{6}}{q^{2}}+\frac{5 M_{V}^{4}}{2}+q^{2} M_{V}^{2}\right] \\
& \left.+\frac{4 c_{d}^{2}}{F^{2}} C_{0}\left(q^{2}, 0,0, M_{S}^{2}\right)\left[\frac{M_{S}^{6}}{q^{2}}+\frac{M_{S}^{4}}{2}\right]\right\} .
\end{aligned}
$$

The three-propagator integral $C_{0}\left(q^{2}, M_{a}^{2}, M_{b}^{2}, M_{c}^{2}\right)$ is defined in appendix A.

\section{$3.5\left\langle v_{\mu} \pi \pi\right\rangle$ 1PI vertex}

The divergences generated by the 1PI loop diagrams shown in Fig. 6 get reabsorbed through the renormalization of the pion wave function $\delta Z_{\pi}$ and the $\mathcal{O}\left(p^{4}\right)$ and $\mathcal{O}\left(p^{6}\right)$ couplings $\tilde{\ell}_{6}$ and $\widetilde{r}_{V 2}$ :

$$
\begin{aligned}
\tilde{\ell}_{6} \equiv \tilde{\ell}_{6}^{r}(\mu)+\delta \tilde{\ell}_{6}(\mu) & ; & \delta \widetilde{\ell}_{6}(\mu)=\left\{3-2 \frac{2 G_{V}^{2}}{F^{2}}+\frac{4 c_{d}^{2}}{F^{2}}\right\} \frac{\lambda_{\infty}}{96 \pi^{2}}, \\
\widetilde{r}_{V 2} \equiv \widetilde{r}_{V 2}^{r}(\mu)+\delta \widetilde{r}_{V 2}(\mu) & ; & \delta \widetilde{r}_{V 2}(\mu)=\frac{F^{2} \lambda_{\infty}}{96 \pi^{2}}\left\{\frac{1}{M_{V}^{2}}+\frac{1}{M_{A}^{2}}\right\} .
\end{aligned}
$$

The resulting finite correction to the lowest-order pion form factor,

$$
\Delta \mathcal{F}\left(q^{2}\right)_{1 \mathrm{PI}}=\Delta \mathcal{F}_{\chi}^{\mathrm{ct}}+\Delta \mathcal{F}_{\chi}^{\mathcal{L}_{2}}+\Delta \mathcal{F}_{\chi}^{V}+\Delta \mathcal{F}_{\chi}^{A}+\Delta \mathcal{F}_{\chi}^{S}+\Delta \mathcal{F}_{\chi}^{P}
$$




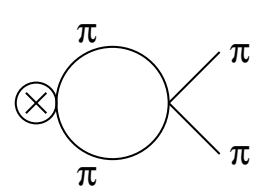

a)

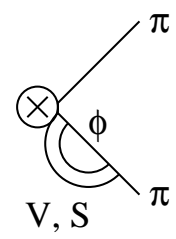

b)

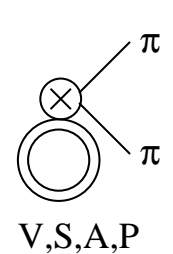

c)

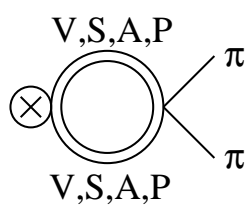

d)

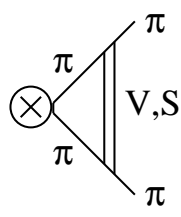

e)

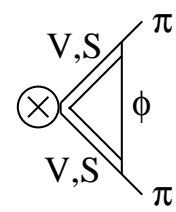

$\delta Z_{\pi}, \tilde{1}_{6}, \tilde{r}_{\mathrm{v}_{2}}$

Figure 6: 1PI diagrams connecting an external vector current and two outgoing pions.

contains contributions from tree-level counterterms,

$$
\begin{aligned}
\Delta \mathcal{F}_{\chi}^{c t}= & -\frac{2 G_{V}^{2}}{F^{2}} \frac{M_{V}^{2}}{16 \pi^{2} F^{2}}\left\{3 \ln \frac{M_{V}^{2}}{\mu^{2}}+\frac{1}{2}\right\}+\frac{4 c_{d}^{2}}{F^{2}} \frac{M_{S}^{2}}{16 \pi^{2} F^{2}}\left\{\ln \frac{M_{S}^{2}}{\mu^{2}}-\frac{1}{2}\right\} \\
& -\widetilde{\ell}_{6}^{r}(\mu) \frac{q^{2}}{F^{2}}+\widetilde{r}_{V 2}^{r}(\mu) \frac{q^{4}}{F^{4}},
\end{aligned}
$$

and loop diagrams with internal Goldstone bosons (first diagram in Fig. 6),

$$
\Delta \mathcal{F}_{\chi}^{\mathcal{L}_{2}}=\frac{q^{2}}{F^{2}}\left\{\frac{1}{6} \hat{B}_{0}\left(q^{2} / \mu^{2}\right)+\frac{1}{144 \pi^{2}}\right\}
$$

and vector,

$$
\begin{aligned}
\Delta \mathcal{F}_{\chi}^{V}= & \frac{2 G_{V}^{2}}{F^{2}} \frac{1}{F^{2}}\left\{-C_{0}\left(q^{2}, 0,0, M_{V}^{2}\right)\left[\frac{M_{V}^{6}}{q^{2}}+\frac{5 M_{V}^{4}}{2}+q^{2} M_{V}^{2}\right]\right. \\
& \left.+C_{0}\left(q^{2}, M_{V}^{2}, M_{V}^{2}, 0\right)\left[\frac{M_{V}^{6}}{q^{2}}+\frac{M_{V}^{4}}{2}\right]-\hat{B}_{0}\left(q^{2} / \mu^{2}\right)\left[\frac{M_{V}^{4}}{q^{2}}+2 M_{V}^{2}+\frac{q^{2}}{12}\right]\right\} \\
& -\frac{\bar{B}_{0}\left(q^{2}, M_{V}^{2}\right)}{F^{2}}\left[\left(2 M_{V}^{2}+\frac{q^{2}}{6}-\frac{q^{4}}{6 M_{V}^{2}}\right)+\frac{2 G_{V}^{2}}{F^{2}}\left(\frac{M_{V}^{4}}{q^{2}}+\frac{2 M_{V}^{2}}{3}-\frac{5 q^{2}}{12}\right)\right] \\
& +\frac{M_{V}^{2}}{16 \pi^{2} F^{2}} \ln \frac{M_{V}^{2}}{\mu^{2}}\left[\left(\frac{q^{2}}{2 M_{V}^{2}}-\frac{q^{4}}{6 M_{V}^{4}}\right)-\frac{2 G_{V}^{2}}{F^{2}}\left(\frac{M_{V}^{2}}{q^{2}}-1+\frac{5 q^{2}}{12 M_{V}^{2}}\right)\right] \\
& +\frac{M_{V}^{2}}{16 \pi^{2} F^{2}}\left[\left(\frac{q^{2}}{2 M_{V}^{2}}-\frac{2 q^{4}}{9 M_{V}^{4}}\right)+\frac{2 G_{V}^{2}}{F^{2}}\left(\frac{M_{V}^{2}}{q^{2}}+1-\frac{19 q^{2}}{36 M_{V}^{2}}\right)\right]
\end{aligned}
$$

axial-vector,

$$
\begin{aligned}
\Delta \mathcal{F}_{\chi}^{A}= & -\frac{\bar{B}_{0}\left(q^{2}, M_{A}^{2}\right)}{F^{2}}\left[2 M_{A}^{2}+\frac{q^{2}}{6}-\frac{q^{4}}{6 M_{A}^{2}}\right]+\frac{M_{A}^{2}}{16 \pi^{2} F^{2}} \ln \frac{M_{A}^{2}}{\mu^{2}}\left[\frac{q^{2}}{2 M_{A}^{2}}-\frac{q^{4}}{6 M_{A}^{4}}\right] \\
& +\frac{q^{2}}{32 \pi^{2} F^{2}}-\frac{q^{4}}{72 \pi^{2} F^{2} M_{A}^{2}},
\end{aligned}
$$


scalar,

$$
\begin{aligned}
\Delta \mathcal{F}_{\chi}^{S}=\frac{4 c_{d}^{2}}{F^{2}} \frac{1}{F^{2}}\left\{-C_{0}\left(q^{2}, 0,0, M_{S}^{2}\right)\left[\frac{M_{S}^{6}}{q^{2}}+\frac{M_{S}^{4}}{2}\right]+C_{0}\left(q^{2}, M_{S}^{2}, M_{S}^{2}, 0\right)\left[\frac{M_{S}^{6}}{q^{2}}-\frac{M_{S}^{4}}{2}\right]\right. \\
\left.\quad-\hat{B}_{0}\left(q^{2} / \mu^{2}\right)\left[\frac{M_{S}^{4}}{q^{2}}+\frac{q^{2}}{12}\right]+\frac{M_{S}^{4}}{16 \pi^{2} q^{2}}\right\}-\frac{q^{2}}{288 \pi^{2} F^{2}}\left[1+\frac{1}{2} \frac{4 c_{d}^{2}}{F^{2}}\right] \\
-\frac{\bar{B}_{0}\left(q^{2}, M_{S}^{2}\right)}{F^{2}}\left[\left(\frac{2 M_{S}^{2}}{3}-\frac{q^{2}}{6}\right)+\frac{4 c_{d}^{2}}{F^{2}}\left(\frac{M_{S}^{4}}{q^{2}}-\frac{M_{S}^{2}}{3}+\frac{q^{2}}{12}\right)\right] \\
-\frac{M_{S}^{2}}{16 \pi^{2} F^{2}} \ln \frac{M_{S}^{2}}{\mu^{2}}\left[\frac{4 c_{d}^{2}}{F^{2}}\left(1+\frac{M_{S}^{2}}{q^{2}}-\frac{q^{2}}{12 M_{S}^{2}}\right)+\frac{q^{2}}{6 M_{S}^{2}}\right]
\end{aligned}
$$

and pseudoscalar resonances,

$$
\Delta \mathcal{F}_{\chi}^{P}=\frac{\bar{B}_{0}\left(q^{2}, M_{P}^{2}\right)}{F^{2}}\left[-\frac{2 M_{P}^{2}}{3}+\frac{q^{2}}{6}\right]-\frac{q^{2}}{96 \pi^{2} F^{2}}\left[\ln \frac{M_{P}^{2}}{\mu^{2}}+\frac{1}{3}\right] .
$$

\section{Vector Form Factor}

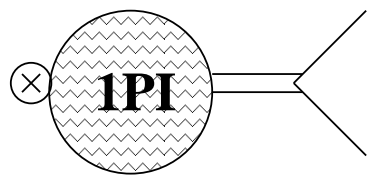

a)

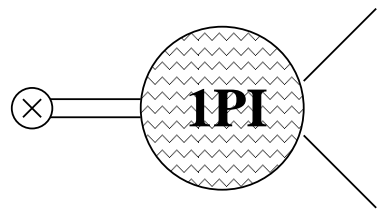

b)

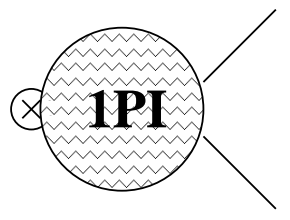

c)

Figure 7: Basic topologies contributing to the Vector Form Factor at NLO.

The basic topologies contributing to the VFF are shown in Fig. [7, in terms of the NLO 1PI diagrams computed in the previous section. The internal $\rho$ line denotes the dressed vector propagator, including the self-energy correction (28) which regulates the $\rho$ pole. Taking this self-energy into account, the LO contribution takes the form:

$$
\mathcal{F}\left(q^{2}\right)_{\mathrm{LO}}=1+\frac{F_{V} G_{V}^{r}(\mu)}{F^{2}} \frac{q^{2}}{M_{V}^{2}-q^{2}-\Sigma_{V}^{r}\left(q^{2}\right)} .
$$

The topology in Fig. 7 7 a generates the following NLO correction:

$$
\Delta \mathcal{F}\left(q^{2}\right)_{F}=\frac{q^{2}}{M_{V}^{2}-q^{2}-\Sigma_{V}^{r}\left(q^{2}\right)} \frac{q^{2}}{F^{2}}\left\{\frac{2 G_{V}^{2}}{F^{2}}\left[\frac{1}{6} \hat{B}_{0}\left(q^{2} / \mu^{2}\right)+\frac{1}{144 \pi^{2}}\right]-2 F \frac{\sqrt{2} G_{V}}{F} X_{F}^{r}(\mu)\right\} .
$$


The second topology (Fig. [7,b) brings the contribution:

$$
\Delta \mathcal{F}\left(q^{2}\right)_{G}=-\frac{q^{2}}{M_{V}^{2}-q^{2}-\Sigma_{V}^{r}\left(q^{2}\right)} \frac{F_{V}}{\sqrt{2} F}\left\{\frac{\sqrt{2} G_{V}}{F} \Delta \Gamma\left(q^{2}, \mu^{2}\right)+\frac{8 X_{G}^{r}(\mu)}{F} q^{2}\right\},
$$

where $\Delta \Gamma\left(q^{2}, \mu^{2}\right)$ is given in Eq. (35). Finally, Fig. 7.c denotes the 1 PI correction $\Delta \mathcal{F}\left(q^{2}\right)_{1 \mathrm{PI}}$ in Eq. (38). Adding all contributions together, one gets the VFF at NLO:

$$
\mathcal{F}\left(q^{2}\right)=\mathcal{F}\left(q^{2}\right)_{\mathrm{LO}}+\Delta \mathcal{F}\left(q^{2}\right)_{F}+\Delta \mathcal{F}\left(q^{2}\right)_{G}+\Delta \mathcal{F}\left(q^{2}\right)_{1 \mathrm{PI}}
$$

Using the large $-N_{C}$ relations (6) in the NLO terms, the result can be written in the form:

$$
\mathcal{F}\left(q^{2}\right)=A\left(q^{2}\right) \frac{M_{V}^{2}}{M_{V}^{2}-q^{2}-\Sigma_{V}^{r}\left(q^{2}\right)}+B\left(q^{2}\right)
$$

where

$$
\begin{aligned}
& A\left(q^{2}\right)=1+\hat{\delta}_{V}+2 M_{V}^{2} \hat{X}-\Delta \tilde{\Gamma}\left(q^{2}\right), \\
& B\left(q^{2}\right)=\mathcal{G}\left(q^{2}\right)-\hat{\delta}_{V}-2\left(M_{V}^{2}+q^{2}\right) \hat{X} .
\end{aligned}
$$

The constants

$$
\begin{aligned}
\hat{\delta}_{V} & \equiv \frac{F_{V} G_{V}^{r}(\mu)}{F^{2}}-1-\Delta \Gamma\left(0, \mu^{2}\right) \\
\hat{X} & \equiv X_{Z}^{r}(\mu)-\frac{1}{F}\left[X_{F}^{r}(\mu)+4 X_{G}^{r}(\mu)\right]
\end{aligned}
$$

and the functions $\Sigma_{V}^{r}\left(q^{2}\right)$,

$$
\Delta \tilde{\Gamma}\left(q^{2}\right) \equiv \Delta \Gamma\left(q^{2}, \mu^{2}\right)-\Delta \Gamma\left(0, \mu^{2}\right)
$$

and

$$
\mathcal{G}\left(q^{2}\right) \equiv \Delta \mathcal{F}\left(q^{2}\right)_{1 \mathrm{PI}}+\Delta \tilde{\Gamma}\left(q^{2}\right) \equiv G\left(q^{2}, \mu^{2}\right)-\Delta \Gamma\left(0, \mu^{2}\right)
$$

are independent of the renormalization scale $\mu$. The subleading $\mathrm{R} \chi \mathrm{T}$ couplings $X_{F}^{r}(\mu)$ and $X_{G}^{r}(\mu)$ only appear through the constant $\hat{X}$, while $X_{Z}^{r}(\mu)$ is also present in the function $\Sigma_{V}^{r}\left(q^{2}\right)$. At $q^{2}=0, \Delta \tilde{\Gamma}(0)=\mathcal{G}(0)=\Sigma_{V}^{r}(0)=0$. Therefore $\mathcal{F}(0)=1$ as it should.

Some 1PI diagrams (Figs. 6, a and 6. e and the V terms in Figs. 6. b and 6. c) have a corresponding reducible counterpart involving a vector propagator. The combination of both contributions can be then incorporated in $A\left(q^{2}\right)$. The function $G\left(q^{2}, \mu^{2}\right)$ contains the corrections generated by the other 1PI diagrams (Figs. 6., d, 6], the S term in Fig. 6. b, the S, A and P terms in Fig. 6. c and the $\widetilde{\ell}_{6}$ and $\widetilde{r}_{V 2}$ pieces in Fig. 6. g). Subtracting their contribution at $q^{2}=0$, which contains the dependence on the renormalization scale $\mu$,

$$
G\left(0, \mu^{2}\right)=\Delta \Gamma\left(0, \mu^{2}\right)=\frac{1}{16 \pi^{2} F^{2}}\left\{M_{V}^{2}\left[\frac{3}{2} \ln \frac{M_{V}^{2}}{\mu^{2}}+\frac{1}{4}\right]+M_{S}^{2}\left[-\ln \frac{M_{S}^{2}}{\mu^{2}}+\frac{1}{2}\right]\right\}
$$


one gets:

$$
\begin{aligned}
\mathcal{G}\left(q^{2}\right)= & \frac{C_{0}\left(q^{2}, M_{V}^{2}, M_{V}^{2}, 0\right)}{F^{2}}\left[\frac{M_{V}^{6}}{q^{2}}+\frac{M_{V}^{4}}{2}\right]+\frac{C_{0}\left(q^{2}, M_{S}^{2}, M_{S}^{2}, 0\right)}{F^{2}}\left[\frac{M_{S}^{6}}{q^{2}}-\frac{M_{S}^{4}}{2}\right] \\
+ & \frac{\bar{B}_{0}\left(q^{2}, M_{V}^{2}\right)}{F^{2}}\left[-\frac{M_{V}^{4}}{q^{2}}-\frac{8 M_{V}^{2}}{3}+\frac{q^{2}}{4}+\frac{q^{4}}{6 M_{V}^{2}}\right]+\frac{\bar{B}_{0}\left(q^{2}, M_{S}^{2}\right)}{F^{2}}\left[-\frac{M_{S}^{4}}{q^{2}}-\frac{M_{S}^{2}}{3}+\frac{q^{2}}{12}\right] \\
+ & \frac{\bar{B}_{0}\left(q^{2}, M_{A}^{2}\right)}{F^{2}}\left[-2 M_{A}^{2}-\frac{q^{2}}{6}+\frac{q^{4}}{6 M_{A}^{2}}\right]+\frac{\bar{B}_{0}\left(q^{2}, M_{P}^{2}\right)}{F^{2}}\left[-\frac{2 M_{P}^{2}}{3}+\frac{q^{2}}{6}\right] \\
+ & \frac{1}{16 \pi^{2} F^{2}}\left\{\frac{M_{V}^{4}+M_{S}^{4}}{q^{2}}+\frac{3}{4} M_{V}^{2}-\frac{1}{4} M_{S}^{2}\right. \\
& +q^{2}\left[\frac{1}{12} \ln \frac{M_{V}^{2}}{\mu^{2}}+\frac{1}{2} \ln \frac{M_{A}^{2}}{\mu^{2}}-\frac{1}{12} \ln \frac{M_{S}^{2}}{\mu^{2}}-\frac{1}{6} \ln \frac{M_{P}^{2}}{\mu^{2}}+\frac{4}{9}-16 \pi^{2} \widetilde{\ell}_{6}^{r}(\mu)\right] \\
& \left.\quad \frac{q^{4}}{6}\left[\frac{1}{M_{V}^{2}} \ln \frac{M_{V}^{2}}{\mu^{2}}+\frac{1}{M_{A}^{2}} \ln \frac{M_{A}^{2}}{\mu^{2}}+\frac{4}{3}\left(\frac{1}{M_{V}^{2}}+\frac{1}{M_{A}^{2}}\right)-\frac{96 \pi^{2}}{F^{2}} \widetilde{r}_{V 2}^{r}(\mu)\right]\right\}
\end{aligned}
$$

\section{Low-Energy Limit}

At very low energies, $q^{2} \ll M_{R}^{2}$, the resonance fields can be integrated out from the effective theory. One recovers then [8] the standard $\chi \mathrm{PT}$ Lagrangian, which leads to the following result for the VFF [35, 36]:

$$
\begin{aligned}
\mathcal{F}_{\chi \mathrm{PT}}\left(q^{2}\right) & =1-\frac{q^{2}}{F^{2}}\left\{\ell_{6}^{r}(\mu)+\frac{1}{96 \pi^{2}}\left[\ln \left(-\frac{q^{2}}{\mu^{2}}\right)-\frac{5}{3}\right]\right\} \\
+ & \frac{q^{4}}{F^{4}}\left\{r_{V 2}^{r}(\mu)+\frac{1}{96 \pi^{2}}\left[\ln \left(-\frac{q^{2}}{\mu^{2}}\right)-\frac{5}{3}\right]\left(2 \ell_{1}^{r}-\ell_{2}^{r}+\ell_{6}^{r}\right)(\mu)+\mathcal{O}\left(\frac{1}{N_{C}^{2}}\right)\right\}+\mathcal{O}\left(\frac{q^{6}}{F^{6}}\right) .
\end{aligned}
$$

The Taylor expansion in powers of $q^{2}$ of the $\mathrm{R} \chi \mathrm{T}$ prediction (49) reproduces the $\chi \mathrm{PT}$ formula (56), as it should. The coefficient of the $\mathcal{O}\left[q^{4} \ln \left(-q^{2} / \mu^{2}\right)\right]$ term satisfies the known large- $N_{C}$ equality $[8,11]\left(2 \ell_{1}-\ell_{2}+\ell_{6}\right)=F^{2}\left(1-5 M_{S}^{2} / M_{V}^{2}\right) /\left(2 M_{S}^{2}\right)$. The non-logarithmic $\mathcal{O}\left(q^{4}\right)$ and $\mathcal{O}\left(q^{6}\right)$ terms relate the low-energy chiral couplings $\ell_{6}$ and $r_{V 2}$ with their $\mathrm{R} \chi \mathrm{T}$ counterparts $\widetilde{\ell}_{6}$ and $\widetilde{r}_{V 2}$ :

$$
\ell_{6}^{r}(\mu)=-\frac{F^{2}}{M_{V}^{2}}\left(1+\hat{\delta}_{V}\right)+\tilde{\ell}_{6}^{r}(\mu)-\frac{1}{96 \pi^{2}}\left[\ln \frac{M_{V}^{2}}{\mu^{2}}-\ln \frac{M_{P}^{2}}{\mu^{2}}+3 \ln \frac{M_{A}^{2}}{\mu^{2}}-\frac{13}{6}\right]
$$




$$
\begin{aligned}
= & -\frac{F_{V} G_{V}^{r}(\mu)}{M_{V}^{2}}+\tilde{\ell}_{6}^{r}(\mu)+\frac{1}{16 \pi^{2}}\left[\frac{4}{3} \ln \frac{M_{V}^{2}}{\mu^{2}}-\frac{1}{2} \ln \frac{M_{A}^{2}}{\mu^{2}}+\frac{1}{6} \ln \frac{M_{P}^{2}}{\mu^{2}}-\frac{M_{S}^{2}}{M_{V}^{2}} \ln \frac{M_{S}^{2}}{\mu^{2}}\right. \\
& \left.+\frac{11}{18}+\frac{M_{S}^{2}}{2 M_{V}^{2}}\right], \\
r_{V 2}^{r}(\mu)= & \frac{F^{2} F_{V} G_{V}^{r}(\mu)}{M_{V}^{4}}+\widetilde{r}_{V 2}^{r}(\mu)+\frac{2 F^{4}}{M_{V}^{2}}\left[\hat{X}-X_{Z}^{r}(\mu)\right] \\
& +\frac{F^{2}}{96 \pi^{2}}\left\{\left(6 \frac{M_{S}^{2}}{M_{V}^{4}}+\frac{1}{2 M_{V}^{2}}-\frac{1}{2 M_{S}^{2}}\right) \ln \frac{M_{S}^{2}}{\mu^{2}}-\frac{9}{M_{V}^{2}} \ln \frac{M_{V}^{2}}{\mu^{2}}-\frac{1}{M_{A}^{2}} \ln \frac{M_{A}^{2}}{\mu^{2}}\right. \\
& \left.\quad-\frac{167}{60 M_{V}^{2}}-\frac{17}{10 M_{A}^{2}}-\frac{3 M_{S}^{2}}{M_{V}^{4}}+\frac{17}{20 M_{S}^{2}}+\frac{1}{10 M_{P}^{2}}\right\} .
\end{aligned}
$$

Notice that the combination of subleading $\mathrm{R} \chi \mathrm{T}$ couplings $\hat{X}$ does not appear at $\mathcal{O}\left(p^{4}\right)$. Therefore, the relation (57) adopts the same form in terms of the effective couplings defined in (19), i.e. $\widetilde{\ell}_{6}^{\text {eff }, r}(\mu)-F_{V}^{\text {eff }} G_{V}^{\text {eff, }, r}(\mu) /\left(M_{V}^{2}\right)^{\text {eff, } r}(\mu)=\widetilde{\ell}_{6}^{r}(\mu)-F_{V} G_{V}^{r}(\mu) / M_{V}^{2}$. As shown in (158), this is no longer true at $\mathcal{O}\left(p^{6}\right)$; nevertheless, the explicit dependence on $\hat{X}-X_{Z}^{r}(\mu)$ present in $r_{V 2}^{r}(\mu)$ can be reabsorbed into the leading term, through the use of the effective couplings, i.e. $r_{V 2}^{r}(\mu)=F^{2} F_{V}^{\text {eff }} G_{V}^{\text {eff, }, r}(\mu) /\left(M_{V}^{4}\right)^{\text {eff }, r}(\mu)+\widetilde{r}_{V 2}^{\text {eff }, r}+\cdots$

Eqs. (57) and (58) contain the well known lowest-order predictions for the two $\chi \mathrm{PT}$ couplings: $\ell_{6}=-M_{V}^{2} r_{V 2}^{r} / F^{2}=-F^{2} / M_{V}^{2}$. Moreover, they give their dependence on the renormalization scale at the NLO. The running of the renormalized couplings $\left[\ell_{6}^{r}(\mu), r_{V 2}^{r}(\mu)\right]$ and $\left[\widetilde{\ell}_{6}^{r}(\mu), \widetilde{r}_{V 2}^{r}(\mu)\right]$ is different, because their corresponding effective theories have a very different particle content.

The $\mu$ dependence of a given coupling "g" can be characterized through the logarithmic derivative

$$
\mu \frac{d g}{d \mu}=-\frac{\gamma_{g}}{16 \pi^{2}} .
$$

From Eqs. (36) and (37) one gets the running of the $\mathrm{R} \chi \mathrm{T}$ couplings:

$$
\gamma_{\widetilde{\ell}_{6}}=\frac{2}{3} \quad, \quad \gamma_{\widetilde{r}_{V 2}}=\frac{F^{2}}{3}\left(\frac{1}{M_{V}^{2}}+\frac{1}{M_{A}^{2}}\right)=\frac{F^{2}}{2 M_{V}^{2}} .
$$

Eqs. (157) and (58) give then the dependence on the renormalization scale of the corresponding $\chi \mathrm{PT}$ couplings:

$$
\gamma_{\ell_{6}}=-\frac{1}{3} \quad, \quad \gamma_{r_{V 2}}=\frac{F^{2}}{6}\left(\frac{5}{M_{V}^{2}}-\frac{1}{M_{S}^{2}}\right) .
$$

These values are in perfect agreement with the low-energy results of refs. $[4,5,36,39]$. The running of the $\mathcal{O}\left(p^{6}\right)$ coupling $r_{V 2}(\mu) / F^{4}$ receives of course additional 2-loop contributions which are of $\mathcal{O}\left(1 / N_{C}^{2}\right)$. 
The rigorous control of the renormalization scale dependences allows us to investigate the successful resonance saturation approximation [8] at the NLO. The $\chi \mathrm{PT}$ couplings $\ell_{6}$ and $r_{V 2}$ have been phenomenologically extracted from a fit to the VFF data at low momenta. This determines [36] the scale-invariant combination

$$
\bar{\ell}_{6} \equiv \frac{32 \pi^{2}}{\gamma_{\ell_{6}}} \ell_{6}^{r}(\mu)-\log \frac{m_{\pi}^{2}}{\mu^{2}}=16.0 \pm 0.5 \pm 0.7,
$$

and

$$
r_{V 2}^{r}\left(M_{\rho}\right)=(1.6 \pm 0.5) \cdot 10^{-4} .
$$

Inserting these numbers in Eqs. (57) and (58), one can estimate the corresponding scaleinvariant combinations of NLO couplings in $\mathrm{R} \chi \mathrm{T}$ :

$$
\begin{gathered}
\hat{\ell}_{6} \equiv \tilde{\ell}_{6}^{r}(\mu)-\frac{\gamma_{\tilde{\ell}_{6}}}{32 \pi^{2}} \log \frac{M_{V}^{2}}{\mu^{2}}-\frac{F^{2}}{M_{V}^{2}} \hat{\delta}_{V}, \\
\hat{r}_{V 2} \equiv \tilde{r}_{V 2}^{r}(\mu)+\frac{F^{4}}{M_{V}^{4}}\left(\hat{\delta}_{V}+2 M_{V}^{2}\left[\hat{X}-X_{Z}^{r}(\mu)\right]\right)-\frac{\gamma_{\widetilde{r}_{V 2}}-\frac{2 F^{4}}{M_{V}^{2}} \gamma_{X_{Z}}}{32 \pi^{2}} \log \frac{M_{V}^{2}}{\mu^{2}},
\end{gathered}
$$

where $\gamma_{X_{Z}}=-1 /\left(6 F^{2}\right)$. Taking $F=92.4 \mathrm{MeV}, M_{V}=770 \mathrm{MeV}$ and $M_{S}=1 \mathrm{GeV}$, one gets $\hat{\ell}_{6}=(-0.2 \pm 0.9) \cdot 10^{-3}$ and $\hat{r}_{V 2}=(-0.2 \pm 0.5) \cdot 10^{-4}$, while a larger value of the scalar resonance mass $M_{S}=1.4 \mathrm{GeV}$ shifts the $\mathcal{O}\left(p^{4}\right)$ coupling to $\hat{l}_{6}=(-0.9 \pm 0.9) \cdot 10^{-3}$, without affecting $\hat{r}_{V 2}$ at the quoted level of accuracy. These numbers should be compared with the large $-N_{C}$ predictions for the $\chi \mathrm{PT}$ couplings $\left.\ell_{6}\right|_{N_{C} \rightarrow \infty}=-F^{2} / M_{V}^{2}=-0.014$ and $\left.r_{V 2}\right|_{N_{C} \rightarrow \infty}=F^{4} / M_{V}^{4}=2.1 \cdot 10^{-4}$. Put in a different way, the hypothesis $\hat{\ell}_{6}=\hat{r}_{V 2}=0$ generates excellent predictions for $\ell_{6}^{r}(\mu)$ and $r_{V 2}^{r}(\mu)$ at any scale $\mu$.

\section{Behaviour at Large Energies}

At large momentum transfer, the relevant renormalization scale invariant functions take the forms:

$$
\begin{gathered}
\mathcal{G}\left(q^{2}\right)=\frac{1}{16 \pi^{2} F^{2}}\left\{-q^{4}\left[\frac{1}{6}\left(\frac{1}{M_{V}^{2}}+\frac{1}{M_{A}^{2}}\right)\left(\ln \frac{-q^{2}}{\mu^{2}}-\frac{2}{3}\right)-\frac{16 \pi^{2}}{F^{2}} \widetilde{r}_{V 2}^{r}(\mu)\right]\right. \\
\left.+q^{2}\left[\frac{1}{3} \ln \frac{-q^{2}}{\mu^{2}}+\frac{16}{9}-16 \pi^{2} \tilde{\ell}_{6}^{r}(\mu)\right]+\mathcal{O}(1)\right\}, \\
\Delta \tilde{\Gamma}\left(q^{2}\right)=\frac{M_{V}^{2}}{16 \pi^{2} F^{2}}\left\{\ln \frac{-q^{2}}{M_{V}^{2}}\left[\ln \frac{q^{2}}{M_{V}^{2}}-2\right]-\frac{1}{2} \ln ^{2} \frac{q^{2}}{M_{V}^{2}}-\frac{\pi^{2}}{6}+\frac{9}{4}+\frac{M_{S}^{2}}{4 M_{V}^{2}}\right\}+\mathcal{O}\left(\frac{1}{q^{2}}\right),
\end{gathered}
$$




$$
\Sigma_{V}^{r}\left(q^{2}\right)=\frac{-q^{4}}{96 \pi^{2} F^{2}}\left\{\ln \frac{-q^{2}}{\mu^{2}}-\frac{5}{3}+192 \pi^{2} F^{2} X_{Z}^{r}(\mu)\right\} .
$$

The $\rho$ propagator makes the $A\left(q^{2}\right)$ piece of the VFF well behaved when $q^{2} \rightarrow \infty$. However, the 1PI contributions generate a wrong behaviour $\mathcal{G}\left(q^{2}\right) \sim q^{4} \ln \left(-q^{2} / \mu^{2}\right)$ in the $B\left(q^{2}\right)$ term, which cannot be eliminated with a local contribution. The problem originates in the tworesonance cut which has an unphysical growing with momenta. Although our leading $\mathrm{R} \chi \mathrm{T}$ Lagrangian (5) only incorporates couplings linear in the resonance fields, the kinetic resonance Lagrangian (4) introduces some bilinear interactions through the chiral connection included in the covariant derivatives. Their couplings are fixed by chiral symmetry and give rise to the 1PI diagrams in Figs. 6. c, 6. d and 6. f. Obviously, these are not the only interactions bilinear in the resonance fields even at large $-N_{C}[17,18,40,41]$. Therefore, it is not surprising that our calculation is unable to find the correct behaviour at large energies for those contributions with two intermediate resonances.

The contributions with an internal vector propagator in diagrams 6 b and 6 , give us some hint about which pieces could be missing in our calculation. These two diagrams combine with a reducible contribution of the type [7,b: the $1 \mathrm{PI}\left\langle V_{\mu \nu} \pi \pi\right\rangle$ vertex in Fig. 5, b. The three contributions contain identical loop functions and their sum generates a global factor $M_{V}^{2} /\left(M_{V}^{2}-q^{2}\right)$, which suppresses the large- $q^{2}$ behaviour. Thus, these corrections have been included in the term $A\left(q^{2}\right)$.

It seems natural to conjecture that the remaining 1PI contributions with two-resonance cuts should combine with the corresponding reducible topologies, including $\langle V R R\rangle$ and $\left\langle v^{\mu} R R\right\rangle$ vertices, to generate the final propagator suppression:

$$
G\left(q^{2}\right) \longrightarrow \frac{M_{V}^{2}}{M_{V}^{2}-q^{2}-\Sigma_{V}^{r}\left(q^{2}\right)} G\left(q^{2}\right) .
$$

The needed Lagrangian takes the form

$$
\mathcal{L}_{V R R}^{2}=i \lambda^{V S S}\left\langle V^{\mu \nu} \nabla_{\mu} S \nabla_{\nu} S\right\rangle+i \lambda^{V P P}\left\langle V^{\mu \nu} \nabla_{\mu} P \nabla_{\nu} P\right\rangle+\cdots
$$

Our conjecture fixes the new chiral couplings in the large $-N_{C}$ limit. It would be interesting to analyze the contributions of this Lagrangian to appropriate Green functions, following the work of refs. [16-18], and check whether the couplings predicted by the corresponding short-distance QCD corrections agree with our naive conjecture. In appendix C, we show two simple examples where the presence of the propagator suppression can be demonstrated in a rather straightforward way.

The behaviour at large energies is also constrained by unitarity requirements. Moreover, the local contributions can be forced to vanish at large $q^{2}$ by taking appropriate values of the $\mathrm{R} \chi \mathrm{T}$ couplings. Probably, this could allow us to determine the scale invariant constants $\hat{\ell}_{6}$ and $\hat{r}_{V 2}$. We plan to investigate all these points in forthcoming works. 


\section{Summary}

The one-loop analysis of the VFF has shown a series of interesting features. As expected, loop diagrams with massive resonance states in the internal lines generate ultraviolet divergences, which require additional higher-dimensional counterterms in the $\mathrm{R} \chi \mathrm{T}$ Lagrangian. Since these counterterms give rise to tree-level contributions which grow too fast at large momenta, their corresponding couplings should be zero at leading order in the large $-N_{C}$ expansion. Thus, one can establish a well defined counting in powers of $1 / N_{C}$ to organize the calculation.

The formal renormalization is completely straightforward at one loop. One can easily determine the $\mu$ dependence of all relevant renormalized couplings. Moreover, the final result is only sensitive to some combinations of the chiral couplings. In fact, using the lowest-order equations of motion, one can eliminate most of the higher-order couplings. Their effects get then reabsorbed into redefinitions of the lowest-order parameters.

Expanding the result in powers of $q^{2} / M_{R}^{2}$, one recovers the usual $\chi \mathrm{PT}$ expression at low momenta. This relates the low-energy chiral couplings $\ell_{6}$ and $r_{V 2}$ with their corresponding $\mathrm{R} \chi \mathrm{T}$ counterparts $\widetilde{\ell}_{6}$ and $\widetilde{r}_{V 2}$. The rigorous control of the renormalization scale dependences has allowed us to investigate the successful resonance saturation approximation at the nextto-leading order in $1 / N_{C}$. The assumption $\hat{\ell}_{6}=\hat{r}_{V 2}=0$ generates excellent predictions for $\ell_{6}^{r}(\mu)$ and $r_{V 2}^{r}(\mu)$ at any scale $\mu$.

At high energies, we have identified a problematic behaviour which originates in the two-resonance cuts: they generate an unphysical increase of the VFF at large values of momentun transfer. This is not surprising, since there are additional contributions generated by interaction terms with several resonances, which have not been included in the minimal $\mathrm{R} \chi \mathrm{T}$ Lagrangian. These new chiral structures should be taken into account to achieve a physical description of the VFF above the two-resonance thresholds. The short-distance QCD constraints can be used to determine their corresponding couplings.

Our calculation represents a first step towards a systematic procedure to evaluate next-toleading order contributions in the $1 / N_{C}$ counting. More work in this direction is in progress.

\section{Acknowledgements}

We have benefited from useful discussions with Oscar Catà, Gerhard Ecker, Santi Peris, Ximo Prades and Jorge Portolés. This work has been supported in part by the EU HPRNCT2002-00311 (EURIDICE), by MCYT (Spain) under grant FPA-2001-3031 and by ERDF funds from the EU Commission. 


\section{Appendix A: Feynman Integrals}

The calculation involves the following Feynman Integrals:

$$
\begin{gathered}
A_{0}\left(M^{2}\right) \equiv \int \frac{d k^{d}}{i(2 \pi)^{d}} \frac{1}{k^{2}+i \epsilon-M^{2}}=-\frac{M^{2}}{16 \pi^{2}}\left[\lambda_{\infty}+\ln \frac{M^{2}}{\mu^{2}}\right], \\
B_{0}\left(q^{2}, M_{a}^{2}, M_{b}^{2}\right) \equiv \int \frac{d k^{d}}{i(2 \pi)^{d}} \frac{1}{\left(k^{2}+i \epsilon-M_{a}^{2}\right)\left[(q-k)^{2}+i \epsilon-M_{b}^{2}\right]} \\
=-\frac{1}{16 \pi^{2}}\left[\lambda_{\infty}+\frac{M_{a}^{2}}{M_{a}^{2}-M_{b}^{2}} \ln \frac{M_{a}^{2}}{\mu^{2}}-\frac{M_{b}^{2}}{M_{a}^{2}-M_{b}^{2}} \ln \frac{M_{b}^{2}}{\mu^{2}}\right]+\bar{J}\left(q^{2}, M_{a}^{2}, M_{b}^{2}\right),
\end{gathered}
$$

and the finite function

$$
\begin{aligned}
& C_{0}\left(q^{2}, M_{a}^{2}, M_{b}^{2}, M_{c}^{2}\right) \equiv \\
& \quad=\int \frac{d k^{d}}{i(2 \pi)^{d}} \frac{1}{\left[\left(p_{1}-k\right)^{2}+i \epsilon-M_{a}^{2}\right]\left[\left(p_{2}+k\right)^{2}+i \epsilon-M_{b}^{2}\right]\left(k^{2}+i \epsilon-M_{c}^{2}\right)},
\end{aligned}
$$

with $q=p_{1}+p_{2}$ and, with massless outgoing pions, $p_{1}^{2}=p_{2}^{2}=0$. The divergences are collected in the factor

$$
\lambda_{\infty}=\frac{2 \mu^{d-4}}{d-4}+\gamma_{E}-\ln 4 \pi-1,
$$

being $\gamma_{E} \simeq 0.5772 \ldots$ the Euler constant and $\mu$ the renormalization scale.

The two-propagator integral contains the finite function

$$
\begin{aligned}
\bar{J}\left(q^{2}, M_{a}^{2}, M_{b}^{2}\right)= & \frac{1}{32 \pi^{2}}\left\{2+\left[\frac{M_{a}^{2}-M_{b}^{2}}{q^{2}}-\frac{M_{a}^{2}+M_{b}^{2}}{M_{a}^{2}-M_{b}^{2}}\right] \ln \frac{M_{b}^{2}}{M_{a}^{2}}\right. \\
& \left.-\frac{\lambda^{1 / 2}\left(q^{2}, M_{a}^{2}, M_{b}^{2}\right)}{q^{2}} \ln \left(\frac{\left[q^{2}+\lambda^{1 / 2}\left(q^{2}, M_{a}^{2}, M_{b}^{2}\right)\right]^{2}-\left(M_{a}^{2}-M_{b}^{2}\right)^{2}}{\left[q^{2}-\lambda^{1 / 2}\left(q^{2}, M_{a}^{2}, M_{b}^{2}\right)\right]^{2}-\left(M_{a}^{2}-M_{b}^{2}\right)^{2}}\right)\right\},
\end{aligned}
$$

with $\lambda(x, y, z) \equiv x^{2}+y^{2}+z^{2}-2 x y-2 x z-2 y z$. Some useful particular cases are:

$$
\begin{aligned}
B_{0}\left(q^{2}, 0,0\right) & =-\frac{\lambda_{\infty}}{16 \pi^{2}}+\hat{B}_{0}\left(q^{2} / \mu^{2}\right), \\
B_{0}\left(q^{2}, M^{2}, M^{2}\right) & =-\frac{1}{16 \pi^{2}}\left\{\lambda_{\infty}+\ln \frac{M^{2}}{\mu^{2}}+1\right\}+\bar{B}_{0}\left(q^{2}, M^{2}\right), \\
B_{0}\left(q^{2}, 0, M^{2}\right) & =-\frac{1}{16 \pi^{2}}\left\{\lambda_{\infty}+\ln \frac{M^{2}}{\mu^{2}}\right\}+\bar{J}\left(q^{2}, 0, M^{2}\right),
\end{aligned}
$$


with the finite parts

$$
\begin{aligned}
\hat{B}_{0}\left(q^{2} / \mu^{2}\right) & =\frac{1}{16 \pi^{2}}\left\{1-\ln \left(-\frac{q^{2}}{\mu^{2}}\right)\right\} \\
\bar{B}_{0}\left(q^{2}, M^{2}\right) & \equiv \bar{J}\left(q^{2}, M^{2}, M^{2}\right)=\frac{1}{16 \pi^{2}}\left\{2-\sigma_{M} \ln \left(\frac{\sigma_{M}+1}{\sigma_{M}-1}\right)\right\}, \\
\bar{J}\left(q^{2}, 0, M^{2}\right) & \equiv \frac{1}{16 \pi^{2}}\left\{1-\left(1-\frac{M^{2}}{q^{2}}\right) \ln \left(1-\frac{q^{2}}{M^{2}}\right)\right\}
\end{aligned}
$$

where $\sigma_{M}=\sqrt{1-4 M^{2} / q^{2}}$.

The relevant three-propagator integrals are:

$$
\begin{aligned}
C_{0}\left(q^{2}, 0,0, M^{2}\right) & =-\frac{1}{16 \pi^{2} q^{2}}\left\{\operatorname{Li}_{2}\left(1+\frac{q^{2}}{M^{2}}\right)-\mathrm{Li}_{2}(1)\right\}, \\
C_{0}\left(q^{2}, M^{2}, M^{2}, 0\right) & =\frac{1}{16 \pi^{2} q^{2}} \ln ^{2}\left(\frac{\sigma_{M}-1}{\sigma_{M}+1}\right),
\end{aligned}
$$

where

$$
\operatorname{Li}_{2}(y) \equiv-\int_{0}^{1} \frac{d x}{x} \ln (1-x y)=-\int_{0}^{y} \frac{d x}{x} \ln (1-x)
$$

is the usual dilogarithmic function.

\section{Appendix B: Lorentz Structures in the Vector Propagators}

In momentum space, the bare vector-field propagator can be written in the form

$$
\left\langle V^{\mu \nu} V^{\rho \sigma}\right\rangle_{0}=\Delta^{\mu \nu, \rho \sigma}(q)=\frac{2 i}{M_{V}^{2}-q^{2}} \mathcal{A}^{\mu \nu, \rho \sigma}(q)+\frac{2 i}{M_{V}^{2}} \Omega^{\mu \nu, \rho \sigma}(q),
$$

with the antisymmetric tensors

$$
\begin{aligned}
\mathcal{A}_{\mu \nu, \rho \sigma}(q) & \equiv \frac{1}{2 q^{2}}\left[g_{\mu \rho} q_{\nu} q_{\sigma}-g_{\rho \nu} q_{\mu} q_{\sigma}-(\rho \leftrightarrow \sigma)\right] \\
\Omega_{\mu \nu, \rho \sigma}(q) & \equiv-\frac{1}{2 q^{2}}\left[g_{\mu \rho} q_{\nu} q_{\sigma}-g_{\rho \nu} q_{\mu} q_{\sigma}-q^{2} g_{\mu \rho} g_{\nu \sigma}-(\rho \leftrightarrow \sigma)\right], \\
\mathcal{I}_{\mu \nu, \rho \sigma} & \equiv \frac{1}{2}\left(g_{\mu \rho} g_{\nu \sigma}-g_{\mu \sigma} g_{\nu \rho}\right),
\end{aligned}
$$

obeying the properties:

$$
\begin{gathered}
\Omega \cdot \mathcal{A}=\mathcal{A} \cdot \Omega=0 \quad, \quad \mathcal{A} \cdot \mathcal{A}=\mathcal{A} \quad, \quad \Omega \cdot \Omega=\Omega \quad, \quad \mathcal{A}+\Omega=\mathcal{I}, \\
q^{\mu} \Omega_{\mu \nu, \rho \sigma}(q)=q^{\nu} \Omega_{\mu \nu, \rho \sigma}(q)=q^{\rho} \Omega_{\mu \nu, \rho \sigma}(q)=q^{\sigma} \Omega_{\mu \nu, \rho \sigma}(q)=0 .
\end{gathered}
$$


For any antisymmetric tensor $H_{\mu \nu, a b}$, the operator $\mathcal{I}_{c d, \alpha \beta}$ acts like the identity, i.e.

$$
H \cdot \mathcal{I}=\mathcal{I} \cdot H=H .
$$

We can then define the antisymmetric inverse $G^{a b, \rho \sigma}$, which satisfies

$$
H_{\mu \nu, a b} G^{a b, \rho \sigma}=G_{\mu \nu, a b} H^{a b, \rho \sigma}=\mathcal{I}_{\mu \nu}^{\rho \sigma} .
$$

The inverse propagator in momentum space is given by

$$
\begin{aligned}
\Delta^{-1}(q)^{\mu \nu, \rho \sigma} & =-i \frac{\left(M_{V}^{2}-q^{2}\right)}{2} \mathcal{A}^{\mu \nu, \rho \sigma}(q)-i \frac{M_{V}^{2}}{2} \Omega^{\mu \nu, \rho \sigma}(q) \\
& =i \frac{q^{2}}{2} \mathcal{A}^{\mu \nu, \rho \sigma}(q)-i \frac{M_{V}^{2}}{2} \mathcal{I}^{\mu \nu, \rho \sigma} .
\end{aligned}
$$

\section{Appendix C: Form Factors with Resonances in the Final State}

We present here a few examples of current matrix elements with external resonances. They show that in order to implement a correct short-distance behaviour, one needs to introduce additional interactions with more than one resonance field. Moreover, the new chiral couplings can be easily determined.

\section{C.1 Axial form factor to $\mathrm{S}_{\mathrm{I}=0}^{0} \pi^{-}$}

Let us consider the two-point correlation function of two axial currents $J_{A}^{\mu}=\bar{d} \gamma^{\mu} \gamma_{5} u$, in the chiral limit:

$$
\Pi_{A A}^{\mu \nu}(q) \equiv i \int d^{4} x \mathrm{e}^{i q x}\left\langle 0\left|T\left(J_{A}^{\mu}(x) J_{A}^{\nu}(0)^{\dagger}\right)\right| 0\right\rangle=\left(-g^{\mu \nu} q^{2}+q^{\mu} q^{\nu}\right) \Pi_{A A}\left(q^{2}\right) .
$$

The associated spectral function $\operatorname{Im} \Pi_{A A}(t)$ is a sum of positive contributions corresponding to the different intermediate states. At large $t$, it behaves as a constant. Therefore, since there is an infinite number of possible states, the absorptive contribution of a given intermediate state should vanish at infinite momentum transfer.

One can easily check that the minimal $\mathrm{R} \chi \mathrm{T}$ Lagrangian of ref. [8], which only contains interactions linear in the resonance fields, generates an absorptive $S_{I=0}^{0} \pi^{-}$contribution with the wrong behaviour at large momenta: $\left.\operatorname{Im} \Pi_{A A}(t)\right|_{S \pi} \sim$ constant. The problem can be easily identified analysing the corresponding form factor, defined through the matrix element

$$
\left\langle S_{I=0}^{0} \pi^{-}\left|\bar{d} \gamma_{\mu} \gamma_{5} u\right| 0\right\rangle=-2 i \mathcal{F}_{S \pi}\left(q^{2}\right)\left(g_{\mu \nu}-\frac{q_{\mu} q_{\nu}}{q^{2}}\right) p_{\pi}^{\nu},
$$




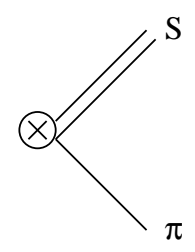

a)

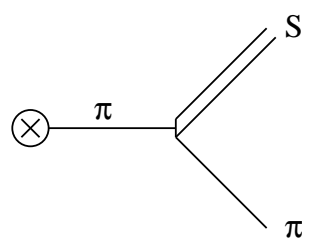

b)

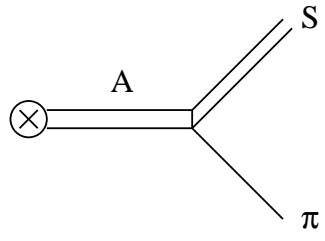

c)

Figure 8: Tree-level contributions to $\mathcal{F}_{S \pi}\left(q^{2}\right)$.

where $q^{\mu}=\left(p_{\pi}+p_{S}\right)^{\mu}$. The lowest-order calculation with the $\mathrm{R} \chi \mathrm{T}$ Lagrangian (diagrams 8, a and 8. b) gives a constant form factor,

$$
\mathcal{F}_{S \pi}\left(q^{2}\right)=\frac{2 c_{d}}{F}
$$

which obviously is not vanishing at infinite momentum transfer.

The correct large energy behaviour can be recovered adding the interaction term [41]

$$
\left.\mathcal{L}_{S A}^{2}=\lambda_{1}^{S A}\left\langle\left\{\nabla^{\mu} S, A_{\mu \nu}\right\} u^{\nu}\right\}\right\rangle
$$

which modifies the form factor (diagram $8, \mathrm{c}$ ):

$$
\mathcal{F}_{S \pi}\left(q^{2}\right)=\frac{2 c_{d}}{F}-\sqrt{2} \lambda_{1}^{S A} \frac{F_{A}}{F} \frac{q^{2}}{M_{A}^{2}-q^{2}} .
$$

Imposing that the form factor must vanish as $q^{2} \rightarrow \infty$, the coupling $\lambda_{1}^{S A}$ is constrained to take the value

$$
\lambda_{1}^{S A}=-\frac{\sqrt{2} c_{d}}{F_{A}}=-\frac{1}{\sqrt{2}} .
$$

The resulting form factor adopts then the usual monopolar form

$$
\mathcal{F}_{S \pi}\left(q^{2}\right)=\frac{M_{A}^{2}}{M_{A}^{2}-q^{2}} .
$$

\section{C.2 Vector form factor to $R_{I=1}^{0} R^{-}(R=S, P)$}

The two-point correlation function of two vector currents has a similar behaviour at short distances. Its spectral function behaves as a constant at large momentum transfer, implying that the form factors associated with each intermediate state should vanish at $q^{2} \rightarrow \infty$.

The minimal $\mathrm{R} \chi \mathrm{T}$ Lagrangian generates the matrix elements:

$$
\left\langle R_{I=1}^{0}\left(p_{1}\right) R^{-}\left(p_{2}\right)\left|\bar{d} \gamma^{\mu} u\right| 0\right\rangle=\sqrt{2}\left(p_{2}-p_{1}\right)^{\mu} \mathcal{F}_{R R}\left(q^{2}\right),
$$




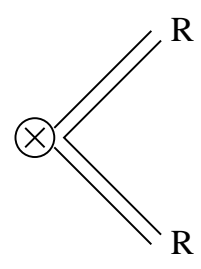

a)

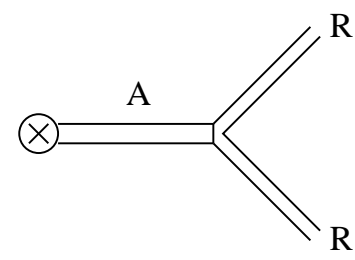

b)

Figure 9: Tree-level contributions to $\mathcal{F}_{R R}\left(q^{2}\right)$.

where $R=S, P$ stands for a scalar or a pseudo-scalar resonance. The corresponding form factors are just constant at lowest order (diagram 9 a):

$$
\mathcal{F}_{R R}\left(q^{2}\right)=1 .
$$

This constant contribution originates in the chiral connection of the resonance kinetic Lagrangians.

It is possible again to recover the right QCD short distance behaviour by adding the interaction terms

$$
\mathcal{L}_{V R R}^{2}=i \lambda^{V R R}\left\langle V^{\mu \nu} \nabla_{\mu} R \nabla_{\nu} R\right\rangle,
$$

which change the form factors to (diagram 9, b)

$$
\mathcal{F}_{R R}\left(q^{2}\right)=1+\frac{F_{V}}{\sqrt{2}} \lambda^{V R R} \frac{q^{2}}{M_{V}^{2}-q^{2}} .
$$

Imposing a proper high energy behaviour one gets the constraint

$$
\lambda^{V R R}=\frac{\sqrt{2}}{F_{V}}=\frac{1}{F},
$$

and a monopolar form for the form factors

$$
\mathcal{F}_{R R}\left(q^{2}\right)=\frac{M_{V}^{2}}{M_{V}^{2}-q^{2}} .
$$

\section{References}

[1] A. Pich, "Aspects of Quantum Chromodynamics", Proc. 1999 ICTP Summer School in Particle Physics (Trieste, Italy, 21 June - 9 July 1999), eds. G. Senjanović and A. Yu. Smirnov, ICTP Series in Theoretical Physics - Vol. 16 (World Scientific, Singapore, 2000) 53 arXiv:hep-ph/0001118. 
[2] A. Pich, "Effective Field Theory", Proc. Les Houches Summer School of Theoretical Physics -Probing the Standard Model of Particle Interactions- (Les Houches, France, 28 July - 5 Sep 1997), eds. R. Gupta et al (Elsevier Science B.V., Amsterdam, 1999), Vol. II, 949 arXiv:hep-ph/9806303.

[3] S. Weinberg, Physica 96A (1979) 327.

[4] J. Gasser and H. Leutwyler, Ann. Phys. 158 (1984) 142.

[5] J. Gasser and H. Leutwyler, Nucl. Phys. B 250 (1985) 465.

[6] G. Ecker, Prog. Part. Nucl. Phys. 35 (1995) 1.

[7] A. Pich, Rep. Prog. Phys. 58 (1995) 563.

[8] G. Ecker, J. Gasser, A. Pich and E. de Rafael, Nucl. Phys. B 321 (1989) 311.

[9] G. Ecker, J. Gasser, H. Leutwyler, A. Pich and E. de Rafael, Phys. Lett. B 223 (1989) 425.

[10] G. 't Hooft, Nucl. Phys. B 72 (1974) 461, 75 (1974) 461; E. Witten, Nucl. Phys. B 160 (1979) 57.

[11] A. Pich, "Colourless Mesons in a Polychromatic World", Proc. Workshop on Phenomenology of Large $-N_{C}$ QCD (Tempe, Arizona, 9-11 January 2002), ed. R.F. Lebed (World Scientific, Singapore 2002) p. 239 arXiv:hep-ph/0205030.

[12] A.V. Manohar, "Large $N_{C}$ QCD", Proc. Les Houches Summer School of Theoretical Physics -Probing the Standard Model of Particle Interactions- (Les Houches, France, 28 July - 5 Sep 1997), eds. R. Gupta et al (Elsevier Science B.V., Amsterdam, 1999), Vol. II, p. 1091 arXiv:hep-ph/9802419.

[13] S. Peris, M. Perrottet and E. de Rafael, JHEP 9805 (1998) 011.

[14] M.F. Golterman and S. Peris, Phys. Rev. D 61 (2000) 034018.

[15] M. Knecht and E. de Rafael, Phys. Lett. B 424 (1998) 335; M. Knecht, S. Peris and E. de Rafael, Phys. Lett. B 443 (1998) 255; M.F. Golterman, S. Peris, B. Phily and E. de Rafael, JHEP 0201 (2002) 024.

[16] M. Knecht and A. Nyffeler, Eur. Phys. J. C 21 (2001) 659.

[17] P.D. Ruiz-Femenía, A. Pich and J. Portolés, JHEP 0307 (2003) 003.

[18] V. Cirigliano, G. Ecker, M. Eidemüller, A. Pich and J. Portolés, arXiv:hep-ph/0404004 [Phys. Lett. B in press].

[19] F. Guerrero and A. Pich, Phys. Lett. B 412 (1997) 382. 
[20] D. Gómez Dumm, A. Pich and J. Portolés, Phys. Rev. D 62 (2000) 054014.

[21] J.J. Sanz-Cillero and A. Pich, Eur. Phys. J. C 27 (2003) 587.

[22] A. Pich and J. Portolés, Phys. Rev. D 63 (2001) 093005.

[23] M. Jamin, J.A. Oller and A. Pich, Nucl. Phys. B 587 (2000) 331.

[24] M. Jamin, J.A. Oller and A. Pich, Nucl. Phys. B 622 (2002) 279.

[25] E. Pallante and A. Pich, Phys. Rev. Lett. 84 (2000) 2568; Nucl. Phys. B 592 (2000) 294;

E. Pallante, A. Pich and I. Scimemi, Nucl. Phys. B 617 (2001) 441.

[26] T.N. Truong, Phys. Rev. Lett. 61 (1988) 2526; A. Dobado, M.J. Herrero and T.N. Truong, Phys. Lett. B 235 (1990) 134; A. Gómez Nicola and J.R. Peláez, Phys. Rev. D 65 (2002) 054009; T. Hannah, Phys. Rev. D 55 (1997) 5613.

[27] J.A. Oller, E. Oset and J.E. Palomar, Phys. Rev. D 63 (2001) 114009; J.A. Oller and E. Oset, Phys. Rev. D 60 (1999) 074023.

[28] O. Catà and S. Peris, Phys. Rev. D 65 (2002) 056014.

[29] M. Bando, T. Kugo, S. Uehara, K. Yamawaki and T. Yanagida, Phys. Rev. Lett. 54 (1985) 1215; M. Bando, T. Kugo and K. Yamawaki, Phys. Rep. 164 (1988) 217; T. Fujiwara, T. Kugo, H. Terao, S. Uehara and K. Yamawaki, Prog. Theor. Phys. 73 (1985) 926.

[30] M. Harada and K. Yamawaki, Phys. Rep. 381 (2003) 1; Phys. Lett. B 297 (1992) 151.

[31] J. Bijnens, P. Gosdzinsky and P. Talavera, Phys. Lett. B 429 (1998) 111; JHEP 9801 (1998) 014; Nucl. Phys. B501 (1997) 495; J. Bijnens and P. Gosdzinsky, Phys. Lett. B 388 (1996) 203.

[32] C.-K. Chow and S.-J. Rey, Nucl. Phys. B 528 (1998) 303; M. Booth, G. Chiladze and A.F. Falk, Phys. Rev. D 55 (1997) 3092.

[33] E. Jenkins, A.V. Manohar and M.B. Wise, Phys. Rev. Lett. 75 (1995) 2272.

[34] A. Pich, J. Portolés, I. Rosell and P. Ruiz-Femenía, work in progress.

[35] J. Gasser and H. Leutwyler, Nucl. Phys. B 250 (1985) 517.

[36] J. Bijnens, G. Colangelo and P. Talavera, JHEP 9805 (1998) 014.

[37] J. Bijnens and P. Talavera, JHEP 0203 (2002) 046.

[38] S. Weinberg, Phys. Rev. Lett. 18 (1967) 507. 
[39] J. Bijnens, G. Colangelo and G. Ecker, JHEP 9902 (1999) 020; Ann. Phys. 280 (2000) 100.

[40] D. Gómez Dumm, A. Pich, and J. Portolés, Phys. Rev. D 69 (2004) 073002.

[41] V. Cirigliano, G. Ecker, M. Eidemüller, R. Kaiser, A. Pich, and J. Portolés, work in progress. 